\title{
Understanding the underlying dimensions in perfumers' odor perception space as a basis for developing meaningful odor maps
}

\author{
Manuel Zarzo \\ Universidad Politécnica de Valencia, Valencia, Spain \\ AND \\ David T. Stanton \\ Procter \& Gamble Company, Cincinnati, Ohio
}

\begin{abstract}
Various low-dimensional perceptual maps of fragrances have been proposed in the literature, as well as sensory maps for the odor descriptors most frequently applied in perfumery. To reach a consensus, however, seems difficult, if at all possible. In the present study, we applied principal components analysis to two databases. The first contains numeric odor profiles of 309 compounds based on 30 descriptors. The loading plot corresponding to the relevant components was strikingly similar to the odor effects diagram proposed by P. Jellinek (1951), primarily on the basis of his long experience as a perfumer. We obtained similar results in our analysis of the second database, which comprises 66 descriptors and contains the semantic descriptions of 119 perfume materials. On the basis of the results of both analyses, a commercial map of fragrances is discussed. Our findings suggest that it is possible to develop standard sensory maps of perfumery odor descriptors, if a consensus is first reached regarding which odorants best represent particular odor qualities.
\end{abstract}

\section{Odor Description and Perception: \\ Basic Concepts}

Various procedures have been proposed for describing odor character (for reviews, see Chastrette, 1998; Wise, Olsson, \& Cain, 2000). Semantic methods are used frequently, because they generate data rapidly. Such methods consist of recording the words that come to mind when one smells a substance. Such words are called odor character descriptors or odor aspect attributes, and usually several are necessary for describing how a scent resembles other common odors.

Another method of odor description consists of using a numeric scale to rate the similarity between a test odor and a series of reference odorants chosen as standards for different descriptors (Schutz, 1964; Yoshida, 1975). A simplified method omits the use of reference materials in order to allow for a larger list of descriptors; instead, odor profiles are obtained by asking individuals to numerically rate attributes that describe an odor's character. Using this procedure, Dravnieks (1985) asked a panel of 120 individuals to smell 138 pure odorant chemicals and score each odorant according to the applicability of 146 descriptors. Because odor descriptions can be influenced by personal experience and subjectivity (Richardson \& Zucco, 1989), the use of a panel is recommended in order to avoid bias in the assignment of odor profiles.
Whether semantic or numeric odor profiles are obtained for a representative set of compounds, the resulting database (i.e., the odorant object space) contains information useful for describing the relationships among compounds on the basis of perceived odorant similarity and dissimilarity. Odor databases are also useful for characterizing odor descriptor space by identifying groups of terms on the basis of their similar and dissimilar meanings; such groupings provide information about how people use different words to characterize odors. When semantic methods are used, two or more descriptors are considered to be similar if they are often applied together to describe a given smell; the opposite applies to dissimilar attributes. When numeric methods are used, two descriptors are considered to be similar if they present a significant positive correlation; they are considered dissimilar if the correlation is negative.

When people are asked to assess the dissimilarity between two odorants on a numeric scale (e.g., $0=i d e n$ tical, $10=$ completely different), rather similar results are expected among the assessors. By contrast, if we ask a panel to rate the woody character of a given odorant, unless a reference material is assigned for this descriptor, it is more difficult to obtain a consensus because of different preconceptions each individual has about the woody odor character. There is no general agreement

M.Zarzo, mazarcas@eio.upv.es 
about which materials should be chosen as references for the odor descriptors used in perfumery (Brud, 1986). Therefore, it seems easier to achieve a consensus in the description of odorant object space. This issue is relevant to perfumers, because part of their work is to determine which odorants should be chosen to develop a successful fragrance.

Despite the problems arising in the characterization of odor descriptor space, the development of standard low-dimensional perceptual maps of odor descriptors is of broad interest in perfumery for several reasons. Such maps (1) clarify consumer preference (Nute, Macfie, \& Greenhoff, 1988), (2) aid in the description of complex mixtures of odorants, (3) enable training of sensory panels, (4) provide certain standards of communication among perfumers, (5) assist in perfume classification (J. S. Jellinek, 1992), and (6) allow better communication among perfume retailers and customers. Actually, with such maps the description of scents becomes easier, because the maps reflect the similarities and dissimilarities among odor categories.

Not all of the terms commonly used to describe a given odor are independent. For example, if a certain substance smells fruity, it is expected that different descriptors, such as apricot, cherry, peach, pineapple, banana, or apple, may be applied to describe the odor character. Because these fruity descriptors share certain similarities, they can be considered an independent odor class. Such similarities among fruity descriptors have been reported in the analysis of a semantic odor database (Zarzo \& Stanton, 2006). From a multivariate standpoint, a class of semantically related attributes can also be regarded as an underlying semantic dimension in the odor descriptor space.

Semantic dimensions are objective constructs, in that they account for odor descriptors that evoke the smell source (e.g., all fruity descriptors recall the scent of one or more fruits). In some cases, underlying dimensions might involve odor descriptors that are semantically very different. A recent study of the Dravnieks database has found that the most salient dimension of odor descriptor space is related to hedonic aspects ( pleasantness) (Zarzo, 2008a). Thus, there is a significant positive correlation among the most pleasant odor descriptors, which are negatively correlated with the unpleasant attributes. Similarly, studies have reported that if a wide range of odorants is assessed, pleasantness is the most salient dimension in descriptions of odorant object space (e.g., Coxon, Gregson, \& Paddick, 1978; Davis, 1979; Schiffman, 1974). Pleasantness is not a semantic dimension, nor is it a dimension of odor character, because it reflects a similarity among descriptors that are semantically very different (i.e., they evoke different things) yet share a pleasant quality and that have clearly different odor characters (e.g., floral, fruity, vanilla).

Odor impressions are sensory experiences, and sometimes we may describe scents by using words associated with sensory perceptions, such as sweet, bitter, and soft. These are subjective descriptors, because they do not clearly evoke a well-defined odor source. Other subjective terms, such as calming, exciting, and exhilarating, are descriptions of odor effects (i.e., physiological and psycho- logical reactions) that may be induced by different senses, including olfaction. Conversely, odors may calm, excite, or exhilarate, but they cannot exert floral, green, fruity, or woody effects on us. Given this distinction between odor sensations and odor effects, it is necessary to define another concept: Dimensions of odor effects are the underlying constructs that comprise odor descriptors that produce a similar effect (e.g., citrus, green, and watery refer to fresh odors, as discussed below, and hence refreshing could be considered to be a dimension of odor effect).

\section{Mapping Perfumery Odor Descriptors: The Odor Effects Diagram}

As a result of his long experience as a perfumer, and also on the basis of empirical evidence, P. Jellinek (1951) developed an olfactory representation that he named the odor effects diagram, which displays contrasting odor effects on opposite corners of a square. This representation relies on the idea that the effects of fragrances and their constituents may be described according to two basic polarities: erogenous versus antierogenous (refreshing) and narcotic versus stimulating. For purposes of clarity, P. Jellinek (1951) inserted the most common descriptors into the odor effects diagram in order to indicate their specific effects. This schematic arrangement is referred to here as Jellinek's odor map. The fourth edition of the original work (P. Jellinek, 1997), available in English, also updates and thoroughly discusses the odor effects diagram (J. S. Jellinek, 1997).

Experimental studies have attempted to verify the effects of odors on the human organism (for a review, see J. S. Jellinek, 1994). Although the erogenous, stimulating, or narcotic effects of individual perfume materials have yet to be proven scientifically, Jellinek's odor map has received remarkable confirmation in some investigations on perfumes. Tisserand (1988) reviewed the use of essential oils as psychotherapeutic agents and found that the odor effects diagram was consistent with his own insights regarding the effects of odors on emotional states. In another study (Thiboud, 1991), a two-dimensional projection of a similarity matrix developed from interviews with consumers in the United States, England, Spain, Brazil, and Japan relating to defined perfume bases and to verbal descriptions also exhibited striking similarities with Jellinek's odor map.

One consumer study used 10 commercial perfumes and obtained free descriptors from female perfume users. A multidimensional scaling of the resulting data found that the two dimensions along which the perfumes were most clearly distinguished were heavy/light and floral/nonfloral (J. S. Jellinek, 1990, 1992). Another consumer study, again with 10 popular fragrances, resulted in a projection in which the dimensions sensual/cool and sensitive/ passionate clearly emerged (J. S. Jellinek, du Bosque, Gschwind, Schubert, \& Scharf, 1992). In both studies, the results were consistent with the basic dimensions of the odor effects diagram.

Additional studies reviewed by J. S. Jellinek (1992, 1997) support the idea that the odor effects diagram is an adequate representation of the psychological impressions 
created by men's and women's commercial fragrances as well as scents used in cosmetics, toiletries, household products, and even the natural scents found in the plant and animal kingdoms. Thus, apart from the advantages of mapping perfumery odor descriptors mentioned above, Jellinek's odor map also facilitates the perfumers' work, in both the creation and sensory evaluation of fragrances. Because Jellinek's odor representation was derived basically from the practical experience of a single author who did not claim absolute exactitude, his map requires further validation and interpretation.

\section{Other Representations of Olfactory Perception Space}

With more than 3,500 perfumes launched since the year 2000 , shopping for a new fragrance can be confusing and frustrating. The perfume-using population needs a perfume classification system based on the way perfumes smell. Perfumery companies have developed different schemes for classifying commercial fragrances: These include the H\&R Genealogy, Analogies of Givaudan (both reproduced by Thiboud, 1991), the Drom fragrance circle (see Brud, 1986), the hexagon of fragrance families (see J. S. Jellinek, 1990), and some others mentioned by J. S. Jellinek (1992). Edwards (2008) has classified more than 5,700 commercial fragrances into 14 categories displayed around a central hub of a sensory map called the Fragrance Wheel. Another sensory wheel of odor descriptors is the Discodor (Harder, 1979). Additional sensory maps of perfume materials have been proposed by firms such as Firmenich, PPF, and Aftelier (2006). However, in most cases, the details of how these odor representations have been developed remain confidential.

Few comprehensive semantic odor-profile databases have been published (Arctander, 1969; Burdock, 2004; Sigma-Aldrich, 2003). Different researchers have numerically coded the semantic descriptions contained in these databases and have analyzed them with multivariate statistical methods (Abe, Kanaya, Komukai, Takahashi, \& Sasaki, 1990; Chastrette, de Saint Laumer, \& Sauvegrain, 1991; Chastrette, Elmouaffek, \& Sauvegrain, 1988; Jaubert, Tapiero, \& Doré, 1995; Madany-Mamlouk, Chee-Ruiter, Hofmann, \& Bower, 2003; Zarzo \& Stanton, 2006). The results led to the classification of descriptors into 10 to 32 clusters, confirming the belief that olfactory perception space is high-dimensional. Callegari, Rouault, and Laffort (1997) compared the information of different odor databases reported in the literature and determined that 25 well-chosen descriptors seemed sufficient to faithfully represent the perceptual olfactory space. Similarly, the Flavornet database (www.flavornet.org) classifies odor descriptors into 25 categories. Another outcome of some of these studies (Abe et al., 1990; Chastrette et al., 1991; Jaubert et al., 1995; Madany-Mamlouk et al., 2003) is the development of two-dimensional representations of odor descriptor space. However, none of them provides a meaningful interpretation for the two dimensions that make up the odor map. Odor profile databases contain useful information for further discussion of the various odor maps reported in the literature, but this issue has not received much attention yet.

Any attempt to reach a consensus in mapping perfumery odor descriptors should start with a detailed study of the odor maps reported in the literature. For this purpose, we analyzed two odor databases of perfume materials with principal components analysis (PCA). In the present article, we discuss the resulting low-dimensional representations of odor descriptor space and compare them with both Jellinek's odor map and the Fragrance Wheel.

\section{METHOD}

\section{The Boelens-Haring Database of Numeric Odor Profiles}

The database obtained by Boelens and Haring (1981) contains 309 compounds assessed by a panel of six perfumers who rated the compounds' similarities to 30 reference materials. Each odor reference was selected to represent the maximum odorous intensity known for a specific odor aspect. The similarities, referred to as odor aspect strengths, were quantified on a 10-point scale, where $0=$ odor aspects not found to be present when smelling a compound, $1=$ minimum noticeable level , and $9=$ undiluted reference substance. The final odor profile of each compound was obtained after averaging the ratings and generating a consensus among the panelists.

Although the panelists who participated in Boelens and Haring's (1981) study disagreed significantly on the odor profile of certain odorants, on the whole they arrived at very comparable profiles for a given compound. We arranged odor profiles from the original publication (Boelens \& Haring, 1981) in a matrix (referred to here as the $B-H$ database) with 309 chemicals (in rows) $\times 30$ variables (in columns). The matrix elements, $x_{i j}$, represent the similarity of the chemical $i$ compared with the odor reference $j$, according to the panel. These variables, which we refer to as odor aspect attributes or simply odor descriptors, are listed in Table 1.

Prior to conducting the statistical analysis of this database, we checked the odor descriptions of the materials in sources such as Brechbill (2007), Green (1999), Müller (1992), Poucher (1974), and Sigma-Aldrich (2003). As a preliminary study, we calculated the correlation coefficient for all possible pairs of descriptors and focused the attention on the 48 highest values. We checked whether a high positive correlation between two descriptors could be partly explained by certain odor similarities of their corresponding reference materials (see the Results and Discussion section for details).

Next, we conducted a PCA using SIMCA-P 10.0 software (www.umetrics.com). Principal components are directions of maximum data variance obtained as linear combinations of the original variables. The projections of observations (odorants, in this case) over these directions are called scores. The contributions of the variables in the formation of a given component are called loadings, $p$ [1] being the loadings in the formation of the first principal component (PC1); $p$ [2], the loadings of $\mathrm{PC} 2$; and so on. A 
Table 1

Odor Description of the 30 Reference Materials in the B-H Database

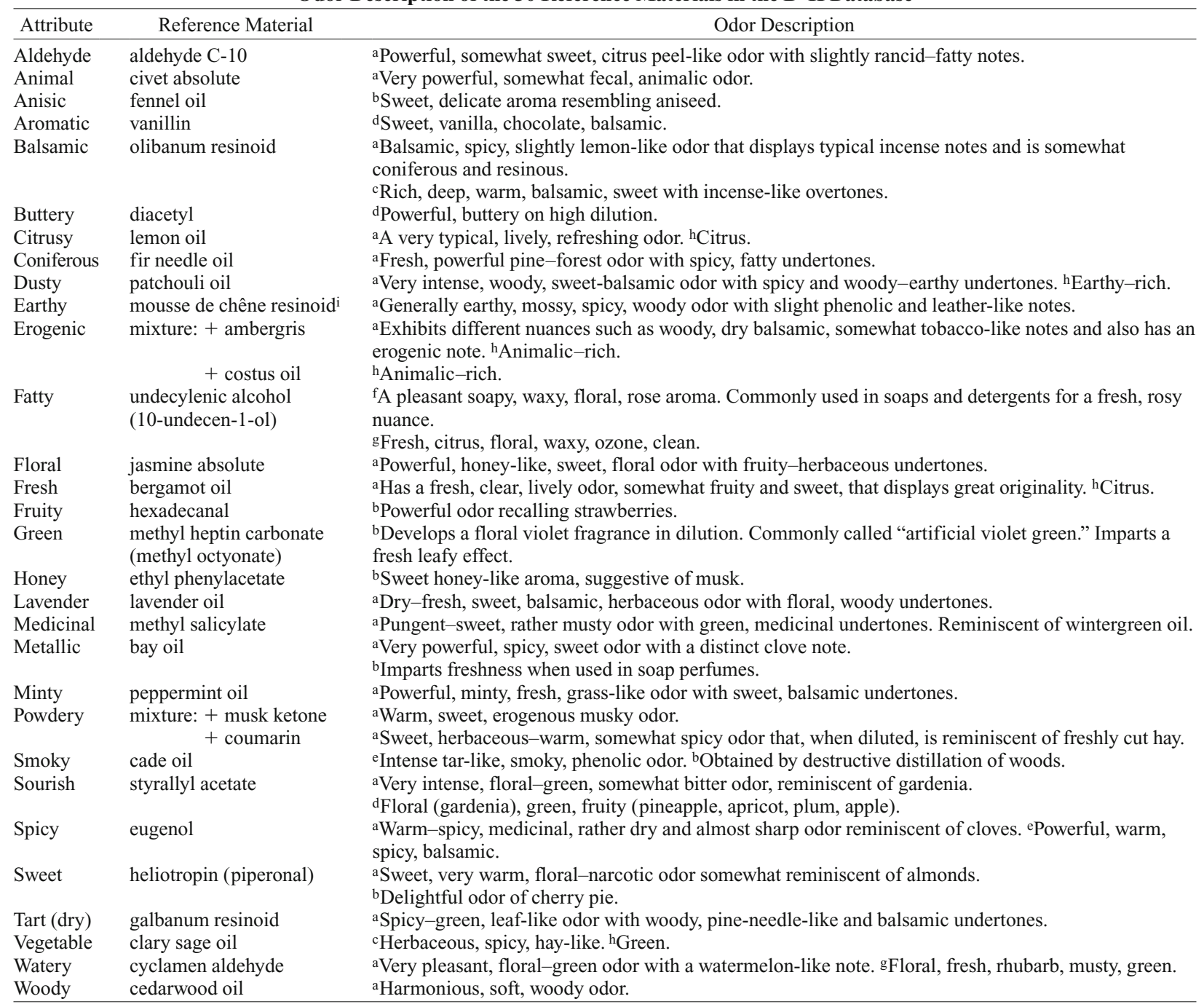

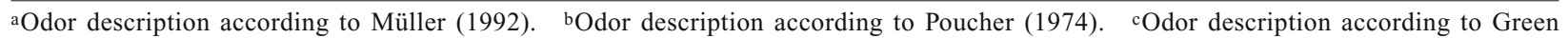
(1999). dOdor description according to Sigma-Aldrich (2003). e Odor description according to Brechbill (2007). fOdor description: www .bedoukian.com/products. gOdor description: www.thegoodscentscompany.com. hClassification of the perfume material according to Aftelier (2006). iFrench name for oakmoss (Aftel, 2001).

scatterplot of the loadings corresponding to two different components is referred to as a loading plot. The loading plot corresponding to $\mathrm{PC} 1$ and $\mathrm{PC} 2$ (i.e., $p[2]$ vs. $p[1]$ ), referred to here as the $P C 1 / P C 2$ plot, usually provides the most relevant information from the database.

The loading plots corresponding to the relevant components were inspected in order to interpret the similarities between attributes, which we discuss according to the odor description of the reference materials as well as the classification of odor descriptors proposed by two reported studies (Abe et al., 1990; Jeltema \& Southwick, 1986). The PC1/PC2 plot was compared with Jellinek's odor map. Attempting to interpret $\mathrm{PC} 1$ and $\mathrm{PC} 2$ as salient dimensions in the perception of perfumery odors, we discuss the results, taking into account the psychological aspects involved in cosmetic odors that were studied extensively by P. Jellinek (1997) and by other authors. For the interpretation of PC2, we also calculated the frequency of occurrence of attributes in the odor description of the fragrances contained in the H\&R Fragrance Guide (Glöss, 1991).

\section{Thiboud's Database of Semantic Odor Profiles}

The second odor database analyzed here was reported by Thiboud (1991). This database contains semantic odor profiles of 119 perfume materials: 44 natural odorants and 75 synthetic chemicals. Each material is labeled with a set of 3 or 4 main odor descriptors and with a number of secondary attributes, ranging from 0 to 14 (with an average of 6.6). The database comprises a total of 85 odor descriptors. The one encountered most frequently is fresh, which is applied as a primary or secondary attribute to $53.8 \%$ of the odorant materials.

In a previous study (Zarzo \& Stanton, 2006), we numerically coded a semantic odor database, which we an- 
Table 2

Forty-Eight Pairs of Odor Aspect Attributes With Highest Correlation

\begin{tabular}{|c|c|c|c|c|c|}
\hline$r$ & Attributes & Common Note & $r$ & Attributes & Common Note \\
\hline .638 & aromatic-sweet & sweet & .394 & balsamic-aromatic & balsamic \\
\hline .628 & tart (dry)-green & green, leafy & .391 & woody-earthy & woody \\
\hline .622 & woody-dusty & woody & .390 & balsamic-powdery & spicy \\
\hline .609 & earthy-dusty & earthy, woody & .380 & smoky-spicy & phenolic \\
\hline .586 & vegetable-tart & spicy, green & .377 & fatty-watery & floral \\
\hline .584 & sourish-fresh & fruity & .375 & anisic-sweet & sweet \\
\hline .571 & earthy-vegetable & spicy & .373 & balsamic-woody & \\
\hline .565 & earthy-tart (dry) & spicy, woody & .371 & watery-fresh & fresh \\
\hline .562 & aldehyde-fatty & fatty & .363 & dusty-spicy & spicy \\
\hline .511 & sourish-green & floral-green & .357 & metallic-tart (dry) & spicy \\
\hline .495 & powdery-erogenic & erogenous & .350 & vegetable-sourish & green \\
\hline .495 & dusty-powdery & spicy & .344 & balsamic-dusty & balsamic, spicy \\
\hline .482 & aldehyde-watery & & .341 & smoky-medicinal & phenolic \\
\hline .466 & smoky-dusty & woody & .338 & balsamic-sweet & sweet \\
\hline .464 & erogenic-animal & animalic & .330 & metallic-watery & fresh \\
\hline .458 & vegetable-green & green & .320 & balsamic-spicy & spicy \\
\hline .454 & smoky-earthy & woody & .316 & minty-fresh & fresh \\
\hline .450 & lavender-fresh & fresh & .312 & lavender-coniferous & fresh \\
\hline .440 & powdery-sweet & sweet, warm & .312 & minty-coniferous & fresh \\
\hline .433 & green-fresh & fresh & .309 & minty-medicinal & sweet \\
\hline .429 & tart-sourish & green & .307 & woody-powdery & \\
\hline .428 & fruity-sourish & fruity & .302 & fruity-fresh & fruity \\
\hline .427 & citrusy-fresh & citrus & .297 & watery-green & floral-green \\
\hline .427 & spicy-aromatic & balsamic & .295 & woody-smoky & woody \\
\hline
\end{tabular}

Note-Common Note $=$ odor note that is supposedly shared in common by both attributes, according to the comparison of odor description for the reference materials (Table 1), as well as from additional sources discussed in the text.

alyzed with PCA. We found that descriptors with fewer than five occurrences did not provide relevant information, and those were discarded. In the present study, we discarded attributes (15 in total) with three or fewer occurrences. Additionally, powerful, weak, and strong were disregarded, because they refer to odor intensity. Harsh and rigorous were considered synonymous and were combined into a single new descriptor, harsh. These adjustments left 66 descriptors for the subsequent multivariate analysis.

The semantic profile of a given odorant was numerically coded by assigning a value of 1 to the descriptors that were applied to describe the primary or secondary odor character of that particular odorant, and 0 otherwise. This procedure yielded an array of 119 odorants $\times$ 66 dichotomic variables, which we called a dichotomic matrix. A second array was created by assigning 1 to the descriptors applied to describe the main odor character of a given odorant, 0.5 to the secondary descriptors, and 0 to the rest. In this case, the 66 variables contain three possible values, and therefore the array was called a trichotomic matrix. Next, a PCA was conducted with each matrix. The relevant loading plots were compared with the $\mathrm{PC} 1 / \mathrm{PC} 2$ plot from the $\mathrm{B}-\mathrm{H}$ database, as well as with Jellinek's odor map, in order to find coincidences and discrepancies.

\section{Comparison Between the Fragrance Wheel and the Odor Effects Diagram}

The Fragrance Wheel displays perfume categories around a central hub, except for aromatic/fougère, which is at the center of the odor wheel (Edwards, 2008). This odor representation was conveniently rotated in order to achieve the best possible coincidence with the odor effects diagram. Taking into account that the narcotic/stimulating dimension of this diagram can be interpreted as feminine versus masculine (P. Jellinek, 1997), we checked the frequency of men's and women's fragrances in each odor category. The results provide clues about how to improve the Fragrance Wheel so that it can better describe the perceptual space of fragrances.

\section{RESULTS AND DISCUSSION}

\section{B-H Database}

Identification of the highest correlation coefficients. The linear correlation coefficient $(r)$ was calculated for all 435 possible pairs of attributes. The correlation was statistically significant $(p<.001)$ in 206 cases. Obviously, this number is unmanageably large for discussing the similarities among descriptors, and so we focused our attention subsequently on only the 48 pairs with highest correlation (Table 2). This information was used to interpret the PCA loading plots.

The highest correlation coefficient $(r=.64)$ corresponds to aromatic and sweet. This similarity is probably caused by a common sweet smell shared by vanillin and heliotropin, which were selected as reference materials for these descriptors (Table 1). Actually, both odorants were regarded by Harper (1975) to be standards for sweet. The extract of the vanilla bean is the best-known example of a sweet-smelling natural product (Müller, 1992). 
Table 3

Most Similar and Dissimilar Odor Aspect Attributes in the B-H Database

\begin{tabular}{|c|c|c|c|c|c|}
\hline Attribute $^{\mathrm{a}}$ & $\overline{\mathrm{x}}_{j}^{\mathrm{b}}$ & Most Similarc & $r_{\max }$ & Most Dissimilard & $r_{\min }$ \\
\hline Fresh & 2.1 & sourish & .58 & powdery & -.58 \\
\hline Sourish & 1.6 & fresh & .58 & powdery & -.44 \\
\hline Citrusy & 0.4 & fresh & .43 & sweet & -.26 \\
\hline Tart (dry) & 1.4 & green & .63 & sweet & -.44 \\
\hline Green & 1.7 & tart (dry) & .63 & sweet & -.41 \\
\hline Vegetable & 1.8 & tart (dry) & .59 & floral & -.27 \\
\hline Metallic & 1.1 & tart (dry) & .36 & sweet & -.28 \\
\hline Watery & 1.7 & aldehyde & .48 & aromatic & -.34 \\
\hline Fatty & 0.6 & aldehyde & .56 & aromatic & -.26 \\
\hline Aldehyde & 0.6 & fatty & .56 & sweet & -.36 \\
\hline Fruity & 1.9 & sourish & .43 & dusty & -.39 \\
\hline Minty & 0.3 & fresh & .32 & floral & -.21 \\
\hline Lavender & 0.3 & fresh & .45 & sweet & -.19 \\
\hline Coniferous & 0.3 & lavender & .31 & floral & -.35 \\
\hline Sweet & 2.2 & aromatic & .64 & $\operatorname{tart}($ dry $)$ & -.44 \\
\hline Aromatic & 1.0 & sweet & .64 & watery & -.34 \\
\hline Honey & 0.3 & aromatic & .27 & fresh & -.13 \\
\hline Anisic & 0.3 & sweet & .37 & watery & -.15 \\
\hline Spicy & 0.8 & aromatic & .43 & watery & -.28 \\
\hline Balsamic & 0.4 & powdery & .39 & fresh & -.37 \\
\hline Powdery & 1.5 & erogenic & .49 & fresh & -.58 \\
\hline Erogenic & 0.4 & powdery & .49 & sourish & -.25 \\
\hline Animal & 0.5 & erogenic & .46 & fresh & -.29 \\
\hline Earthy & 1.1 & dusty & .61 & fruity & -.34 \\
\hline Dusty & 1.3 & woody & .62 & fruity & -.39 \\
\hline Woody & 1.0 & dusty & .62 & fruity & -.22 \\
\hline Smoky & 0.2 & dusty & .47 & fruity & -.25 \\
\hline Medicinal & 0.6 & smoky & .34 & fruity & -.21 \\
\hline Buttery & 0.5 & fruity & .19 & dusty & -.18 \\
\hline Floral & 3.1 & sweet & .28 & conifer & -.35 \\
\hline
\end{tabular}

a Odor aspect attributes, sorted properly in the table to provide an easier interpretation. bAverage value for the 309 compounds. cMost similar attribute (highest positive correlation coefficient). dMost dissimilar attribute (highest negative correlation coefficient).

Odor descriptions were also checked for the remaining 47 attribute pairs with highest correlation, and some shared notes were found in most cases (Table 2). The correlation between fresh and citrusy $(r=.43)$ is rather obvious because the reference materials of both descriptors are obtained from the peel of citrus fruits, and different authors have classified these materials as citrus (Aftelier, 2006; Harper, 1975). Vegetable and tart (dry) are also correlated $(r=.59)$, and the references assigned to both attributes were classified as green (Aftelier, 2006). Patchouli oil was the selected reference for dusty, but Aftelier classifies it as earthy, which explains the strong correlation observed between dusty and earthy $(r=.61)$.

Next, the highest positive and negative correlation coefficients were identified for each attribute (Table 3). An examination of the results showed that most of the first 14 attributes in Table 3 were similar to fresh or to related notes (sourish, tart, aldehyde) and were dissimilar to sweet, aromatic, or powdery. Curiously, the opposite occurred for most of the remaining attributes. We examined this observation in more detail as part of the subsequent multivariate analysis.

PCA. If PCA is applied directly to a given matrix with no data pretreatment, $\mathrm{PC} 1$ provides information about the mean profile of all observations and allows for the identification of the variables with the highest averages.
If a PCA is conducted with centered data (subtracting the mean odor profile so that all centered variables have a null average), the subsequent principal components are influenced by the variables with highest variances. Because all variables in the present study were measured using the same numeric scale (0-9), the highest variance corresponds in general with the highest average. PCA applied to the mean-centered $\mathrm{B}-\mathrm{H}$ database yielded a $\mathrm{PC} 1 / \mathrm{PC} 2$ plot (Figure 1) in which all variables outside of the central cluster have an average $\geq 1$ (average values shown in parentheses), with the exception of metallic. This loading plot provides information about the general data variability, but here the main interest is to study the correlation structures among variables. It is therefore more appropriate to use the autoscaling pretreatment (i.e., variables mean centered and scaled to unit variance) in order to prevent PCs from being influenced by the variables with the highest variance. If this pretreatment is used, the $\mathrm{PC} 1 / \mathrm{PC} 2$ plot undergoes a dramatic change (Figure 2).

One approach commonly used in PCA that was also adopted in another reported analysis of this database (Ennis, Boelens, Haring, \& Bowman, 1982) is to focus on PCs with an eigenvalue of $>1$. This criterion is satisfied by PC1 and by further components up to PC9 (Table 4), so it seemed reasonable to study the loading plots for these components. Another approach is based on the amount of variance explained by cross-validation $\left(Q^{2}\right)$. PCA following the autoscaling pretreatment option indicated that PC1 and PC2 are the only components that satisfy the cross-validation criterion, because their $Q^{2}$ value is higher than the threshold considered by the software SIMCA-P 10.0. Nonetheless, the $Q^{2}$ values of PC3, PC4, and PC6 are close to the threshold (Table 4). PC1 and PC2 account for $32 \%$ of the total data variance, and hence the $\mathrm{PC} 1 /$ PC2 plot (Figure 2) may be expected to provide the most relevant information.

Twelve odor descriptors in the $\mathrm{B}-\mathrm{H}$ database have a score of 0 for more than $75 \%$ of the odorants. These descriptors basically correspond to the attributes in Table 3 with the lowest average value. To determine the effect of these descriptors in the resulting loading plot, these 12 variables were discarded, and a new PCA was conducted with the autoscaling pretreatment. The resulting PC1/PC2 plot was very similar to Figure 2, although it appeared slightly rotated. Another PCA was carried out after centering the data, and the plot was nearly the same as that in Figure 1. Hence, the descriptors rated with a low frequency did not introduce noise in the results.

In an attempt to ease the interpretation of similarities between descriptors, in Figure 2, we highlight the pairs of variables with highest correlation shown in Table 2 . The results reveal that very few variables with $p[1]<0$ are strongly associated with those characterized by $p[1]>0$. This categorization of attributes according to the $p$ [1] loadings is also reflected by checking the most similar and most dissimilar descriptors for each attribute (Table 3). Actually, for all attributes with $p[1]>0$, the most similar descriptor also presents a positive $p$ [1], but it presents a negative $p[1]$ for the most dissimilar. Thus, PC1 provides a gross classification of odor descriptors 


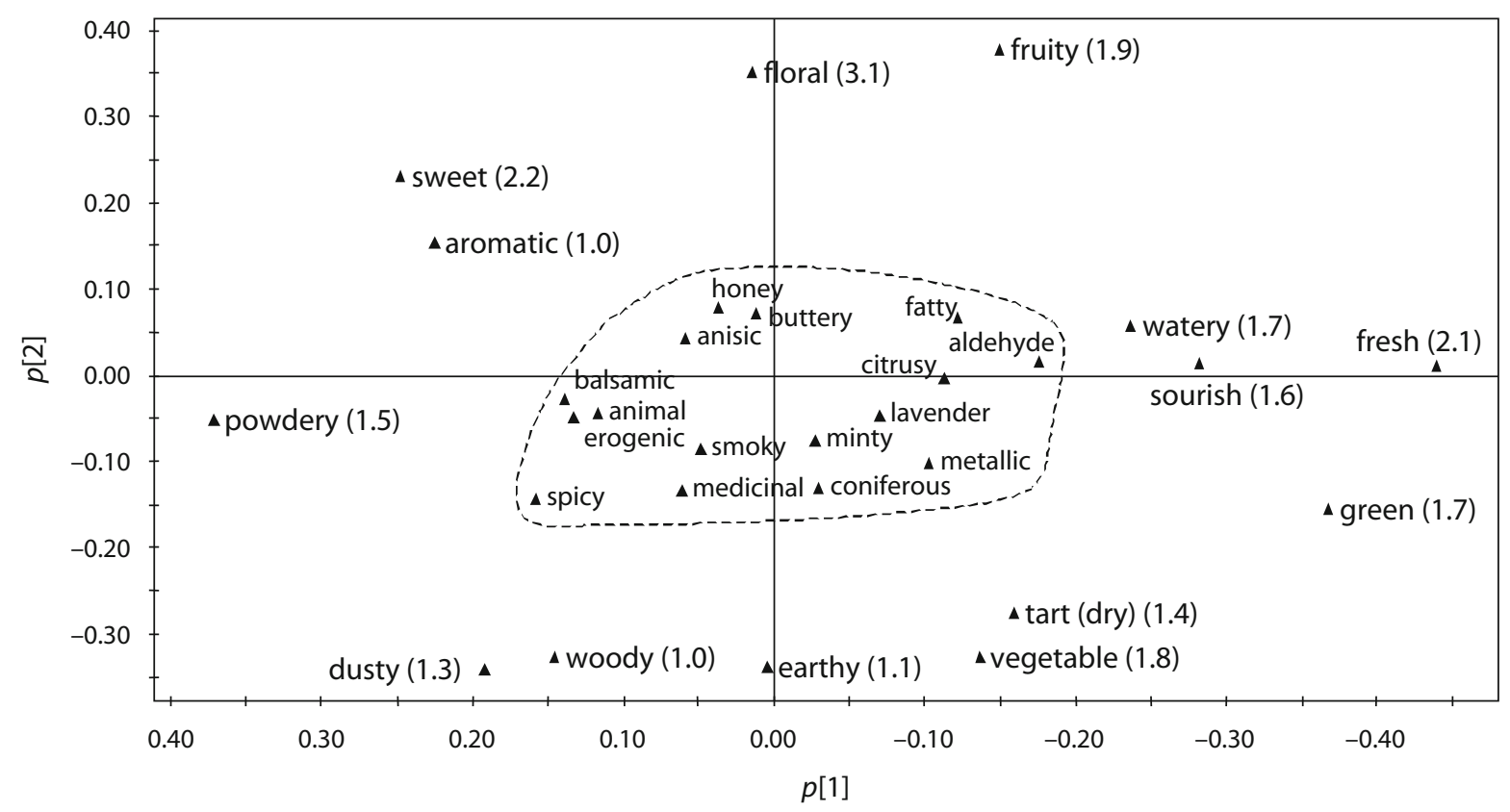

Figure 1. Loading plot for the first and second principal components (PC1/PC2) from the PCA conducted with the B-H database after centering the variables. Numbers in brackets correspond to the average value of each descriptor according to Table 3.

according to a certain underlying dimension of odor perception.

Most odor attributes of the B-H database are included in the long list of descriptors classified by Abe et al. (1990) and Jeltema and Southwick (1986) shown in Table 5. Although a reasonable agreement occurs in the classification of many of these descriptors, some discrepancies are apparent (e.g., lavender, fatty, or metallic); these are discussed in detail below. Jeltema and Southwick analyzed a database of 415 odorants assessed by a panel of about 20 individuals according to the applicability of 146 odor descriptors. A factorial analysis of this database resulted in the classification of these descriptors into 17 groups. Abe et al. selected 126 odor descriptors for $1,573 \mathrm{com}$ -

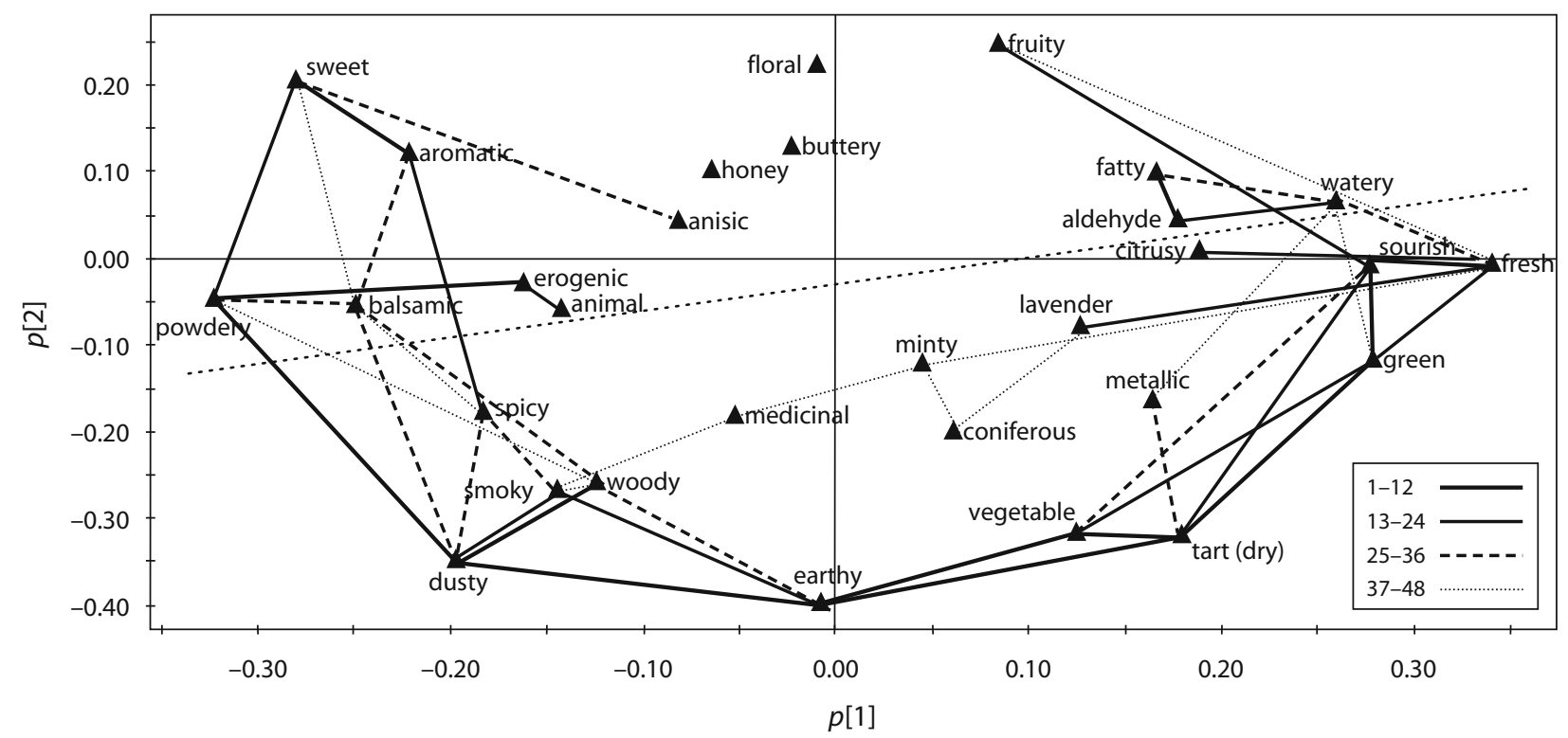

Figure 2. Loading plot for PC1/PC2 from the PCA conducted with the B-H database.Variables were autoscaled prior to the analysis. The 48 pairs of odor descriptors with highest correlation coefficient indicated in Table 2 are highlighted: Thicker solid lines correspond to the 12 highest values, thinner solid ones indicate the next 12 highest values, and so on, according to the legend shown in the figure. Descriptors above the dotted line are more frequently encountered in feminine than in masculine fragrances (Tables 6 and 7). 
Table 4

Summary Overview of Two PCA Models for 10 PCs With Autoscaling Pretreatment

\begin{tabular}{|c|c|c|c|c|c|c|c|c|c|c|}
\hline \multirow[b]{2}{*}{$\underline{\mathrm{PC}}$} & \multicolumn{5}{|c|}{ PCA of the B-H Database } & \multicolumn{5}{|c|}{ PCA of Thiboud's Database } \\
\hline & $R_{x}^{2}$ & $R_{\text {cum }}^{2}$ & Eigenvalue & $Q^{2}$ & $Q^{2}{ }_{\text {limit }}$ & $R_{x}^{2}$ & $R_{\text {cum }}^{2}$ & Eigenvalue & $Q^{2}$ & $Q^{2}$ limit \\
\hline 1 & .175 & .175 & 5.25 & .102 & .035 & .105 & .105 & 6.94 & .048 & .023 \\
\hline 2 & .142 & .317 & 4.26 & .106 & .036 & .090 & .195 & 5.92 & .046 & .023 \\
\hline 3 & .084 & .401 & 2.53 & .030 & .038 & .056 & .251 & 3.70 & .002 & .024 \\
\hline 4 & .066 & .467 & 1.98 & .011 & .039 & .050 & .301 & 3.27 & -.001 & .024 \\
\hline 5 & .058 & .525 & 1.73 & -.030 & .040 & .044 & .344 & 2.90 & -.011 & .024 \\
\hline 6 & .054 & .579 & 1.63 & .020 & .042 & .038 & .383 & 2.51 & -.023 & .025 \\
\hline 7 & .044 & .623 & 1.32 & -.035 & .043 & .036 & .418 & 2.37 & -.022 & .025 \\
\hline 8 & .036 & .659 & 1.07 & -.066 & .045 & .035 & .453 & 2.30 & -.019 & .025 \\
\hline 9 & .034 & .693 & 1.02 & -.050 & .047 & .031 & .484 & 2.06 & -.027 & .026 \\
\hline 10 & .032 & .724 & 0.94 & -.055 & .049 & .027 & .512 & 1.80 & -.037 & .026 \\
\hline
\end{tabular}

Note- $R_{x}^{2}$, variance explained; $R_{\text {cum }}^{2}$, cumulated values; $Q^{2}$, variance explained by cross-validation; $Q^{2}$ limit, threshold value for $Q^{2}$ to satisfy the cross-validation criterion.

pounds from Arctander's (1969) database and applied cluster analysis, which allowed classification of these descriptors into 19 clusters. Arctander's database was compiled in the context of perfumery, and most odors were characterized by only one person (S. Arctander), resulting in an arguable degree of personal subjectivity. Maybe for this reason, a recent study (Pintore et al., 2006) has found significant differences between Arctander's database and another commercial database of semantic odor profiles (BACIS, 2001).
The descriptors anisic, buttery, and honey are closest to the center of the loading plot in Figure 2, revealing a low correlation with the rest of the variables. Because of this, these three descriptors may be expected to form orthogonal directions of variability. Actually, these attributes present the highest loadings in PCs 7, 9, and 10 (figures not shown). The $r_{\max }$ and $\left|r_{\min }\right|$ of these descriptors are among the lowest in Table 3 , confirming their low correlations with the rest of the variables. Consistent with these results, anise was proposed as an independent odor

Table 5

Classification of Odor Attributes Contained in the B-H Database

\begin{tabular}{|c|c|c|c|c|c|}
\hline \multirow{2}{*}{$\begin{array}{l}\text { B-H Odor } \\
\text { Attribute }^{\mathrm{a}}\end{array}$} & \multicolumn{2}{|r|}{ Classification According to Abe et al. (1990) } & \multicolumn{3}{|c|}{ Classification of Jeltema and Southwick (1986) } \\
\hline & $\mathrm{CN}^{\mathrm{b}}$ & Subcluster and Individual Descriptors ${ }^{\mathrm{c}}$ & Dravnieks Descriptor $^{d}$ & $\mathrm{FNe}^{\mathrm{e}}$ & Factor Name ${ }^{f}$ \\
\hline Balsamic & 1 & Balsamic (amber, oriental) & & & \\
\hline Spicy & 1 & Spicy (cinnamic) & Spicy & 13 & spicy \\
\hline Aromatic & 1 & vanillin: intermediate of Spicy and Balsamic & Vanilla & $6-14$ & intermediate brown-almond \\
\hline Floral & 2 & $\begin{array}{l}\text { Floral (hyacinth, heliotrope, narcissus, lily, mimosa, } \\
\text { lilac ...) }\end{array}$ & Floral & 7 & floral \\
\hline Anisic & 3 & Anise (fennel) & Anise & 17 & caraway, anise \\
\hline Vegetable & 4 & Herbaceous (tobacco, hay, tea, lavender, clary-sage . . .) & Fresh green vegetable & 4 & green \\
\hline Lavender & 4 & individual descriptor within Herbaceous & Lavender & 7 & floral \\
\hline Fruity & 6 & $\begin{array}{l}\text { Fruity (apple, pineapple, banana, jasmine, apricot, } \\
\text { plum ...) }\end{array}$ & Non-citrus fruit & 3 & non-citrus fruit \\
\hline Buttery & 7 & [?] (buttery, creamy, berry) & Buttery & 6 & brown \\
\hline Citrusy & 9 & Citrusy (lemony) & Citrus & 10 & citrus \\
\hline Fresh & 9 & [?] (bergamot) & & & \\
\hline Fatty & 11 & Fatty (rancid) & Oily, fatty & 1 & animal, foul \\
\hline Green & 12 & $\begin{array}{l}\text { Green (leafy, vegetable, metallic, violet-leaf, } \\
\text { cucumber ...) }\end{array}$ & Green & 4 & green \\
\hline Metallic & 12 & individual descriptor within Green & Metallic & 2 & solvent \\
\hline Animal & 13 & Animal (civet) & Animal & 1 & animal, foul \\
\hline Honey & 13 & Honey (honey) & Honey & 6 & brown \\
\hline Aldehyde & 14 & [?] (aldehyde) & Alcoholic & 2 & solvent \\
\hline Woody & 15 & Woody (peppery, sap, bark) & Woody & 12 & woody \\
\hline Minty & 15 & Minty (minty) & Minty & 8 & cool, minty \\
\hline Coniferous & 15 & [?] (orrisy, pine) & Turpentine & 2 & solvent \\
\hline Earthy & 16 & Earthy (mossy, root, walnut) & Musty, earthy, moldy & 4 & green \\
\hline Medicinal & 17 & Medicinal (phenolic) & Medicinal & $2-8$ & intermedite solvent-minty \\
\hline Smoky & 17 & [?] (tar, leather, smoky) & Burnt, smoky & $5-9$ & intermediate nutty-burnt \\
\hline Sourish & & & Sour, vinegar & 1 & animal, foul \\
\hline Sweet & & & Sweet & 3 & non-citrus fruit \\
\hline
\end{tabular}

a Some descriptors with an uncertain classification were omitted. bCluster number. Descriptors grouped under the same cluster are supposed to account for similar odors. ' cMost clusters proposed by Abe et al. (1990) are divided in up to four subclusters, and each one comprises a set of different individual odor descriptors (indicated within parentheses). dDravnieks descriptor that is supposed to be most similar to each attribute in the B-H database. eFactor number. Descriptors grouped under the same factor are supposed to account for similar odors. ${ }^{\mathrm{f}}$ Intermediate indicates classification in two categories. 
A

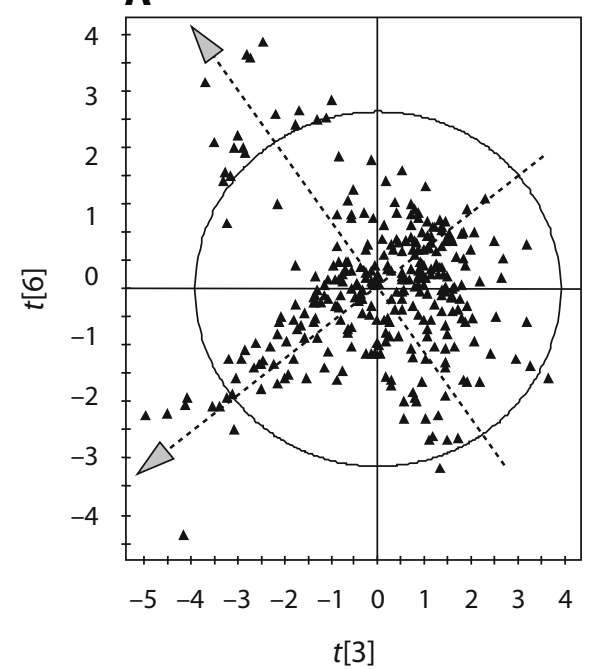

B

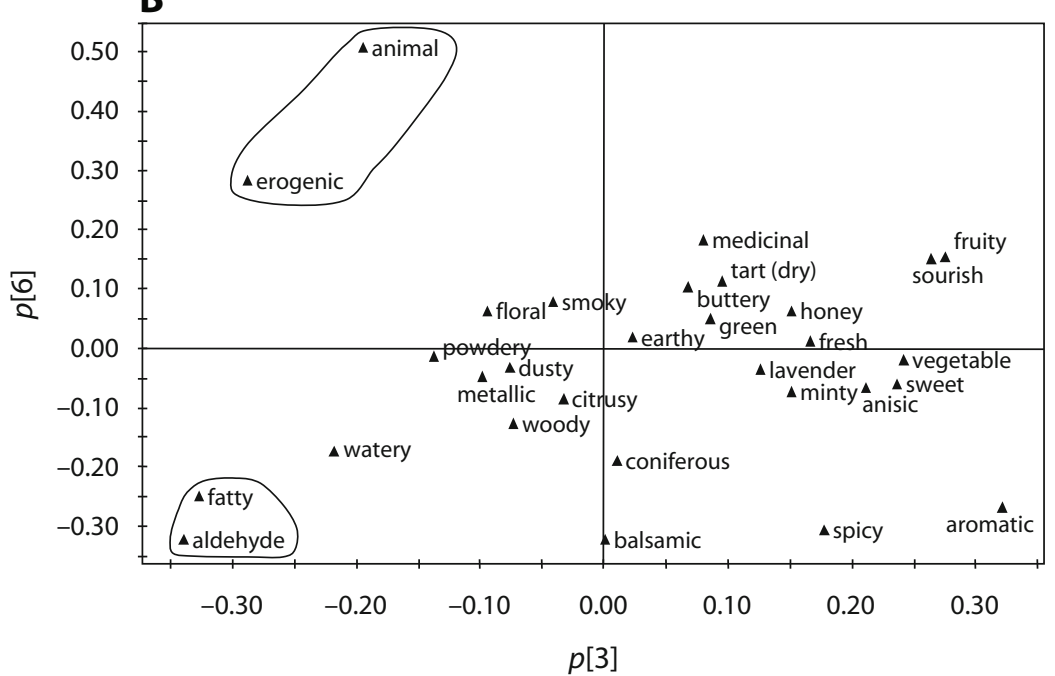

Figure 3. (A) Score plot (t[6] vs. $t[3])$ and (B) loading plot ( $p[6]$ vs. $p[3])$ corresponding to PC3/PC6 from the PCA conducted with the $B-H$ database. Variables were autoscaled prior to the analysis.

by several authors (e.g., Jennings-White, 1984; Rimmel, 1895), and this criterion has been corroborated by the statistical analyses of odor profile databases (Table 5). In the case of buttery, various authors have regarded it as a rather independent descriptor (e.g., Abe et al., 1990; Zarzo \& Stanton, 2006). In contrast, Jeltema and Southwick (1986) classified it as brown (a category that groups balsamic-related odors), but the correlation butterybalsamic is not statistically significant $(p=.73)$ in the $\mathrm{B}-\mathrm{H}$ database.

Honey scents are described as sweet, heavy, and syrupy, with a waxy background (Thiboud, 1991). The same criterion was used in the Sigma-Aldrich (2003) catalog of flavors and fragrances, because honey, sweet, vanilla, and caramel (i.e., syrupy odors) are classified as balsamic. Similarly, Jeltema and Southwick (1986) regarded honey as a brown descriptor (Table 5). Consistent with these similarities, honey yields the highest correlation with sweet and aromatic (i.e., vanillin-like) in the B-H database. By contrast, honey and animal were classified by Abe et al. (1990) in the same cluster (Table 5), but the correlation between both descriptors is not statistically significant ( $p=.54)$.

Interpretation of $\mathrm{PC} 3$ and further principal components. Attempting to further understand the similarities between descriptors, we inspected different loading plots for $\mathrm{PC}_{i} / \mathrm{PC}_{j}(i, j$ from 3 to 10$)$. The score plot for PC3/PC6 (Figure 3, left) shows two rotated directions of variability, and the corresponding loading plot (Figure 3, right) clearly reveals the variables responsible for those directions. One of them is determined by animal and erogenic, suggesting that these descriptors are similar. Actually, the reference materials for both attributes were classified by Aftelier (2006) as animalic-rich. The score plot also suggests that fewer than 30 odorants in the B-H database account for this animal-erogenic dimension. Because of this low number of occurrences, the resulting average value of the two descriptors is low (Table 3 ).
Although a zero score was used often, the similarity between both attributes is reflected clearly in Figure 3, and it is consistent with the criterion of perfumers, as commented on below. Moreover, the correlation coefficient between them is the 15 th highest value among all possible descriptor pairs (Table 2). The same discussion applies to other descriptors in the $\mathrm{B}-\mathrm{H}$ database rated with a low frequency.

Another direction of variability is determined by aldehyde and fatty (Figure 3). Both attributes are correlated $(r=.56)$, probably because decanal (the reference for aldehyde in the $\mathrm{B}-\mathrm{H}$ database) displays a fatty note (Table 1). The similarity between both descriptors is also deduced from a poll of 120 perfumers (Brud, 1986), 18\% of whom chose different aldehydes as reference materials for fatty. Watery appears in Figure 3 very close to fatty and aldehyde. The similarity among these descriptors was also reported by Müller (1992), who described the odor effect produced by short-chain aliphatic aldehydes as "fatty," "watery," and "tallowy," or even "snuffed candle."

Fatty is usually used to describe smells that suggest oil, lard, or wax (Müller, 1992). In a study of Arctander's (1969) database, a cluster was formed with fatty, oily, and waxy (Chastrette et al., 1988). In the context of perfumery, a given odor may be described as fatty if it presents a certain oily, waxy, or rancid note, although other aspects, such as floral or green, might predominate.

Medicinal is the descriptor with highest contribution in PC5. It yields the highest correlation with smoky and minty (Table 2). Thus, it appears in Figure 2 in the middle of both descriptors, and close to minty in Figure 4. Abe et al. (1990) also regarded medicinal and smoky as similar. The similarity between medicinal and minty was also reported by Jeltema and Southwick (1986) (Table 5). Taking into account that phenolic and medicinal are related descriptors (Abe et al., 1990; Chastrette et al., 1988), the correlation medicinal-smoky can be explained by the 

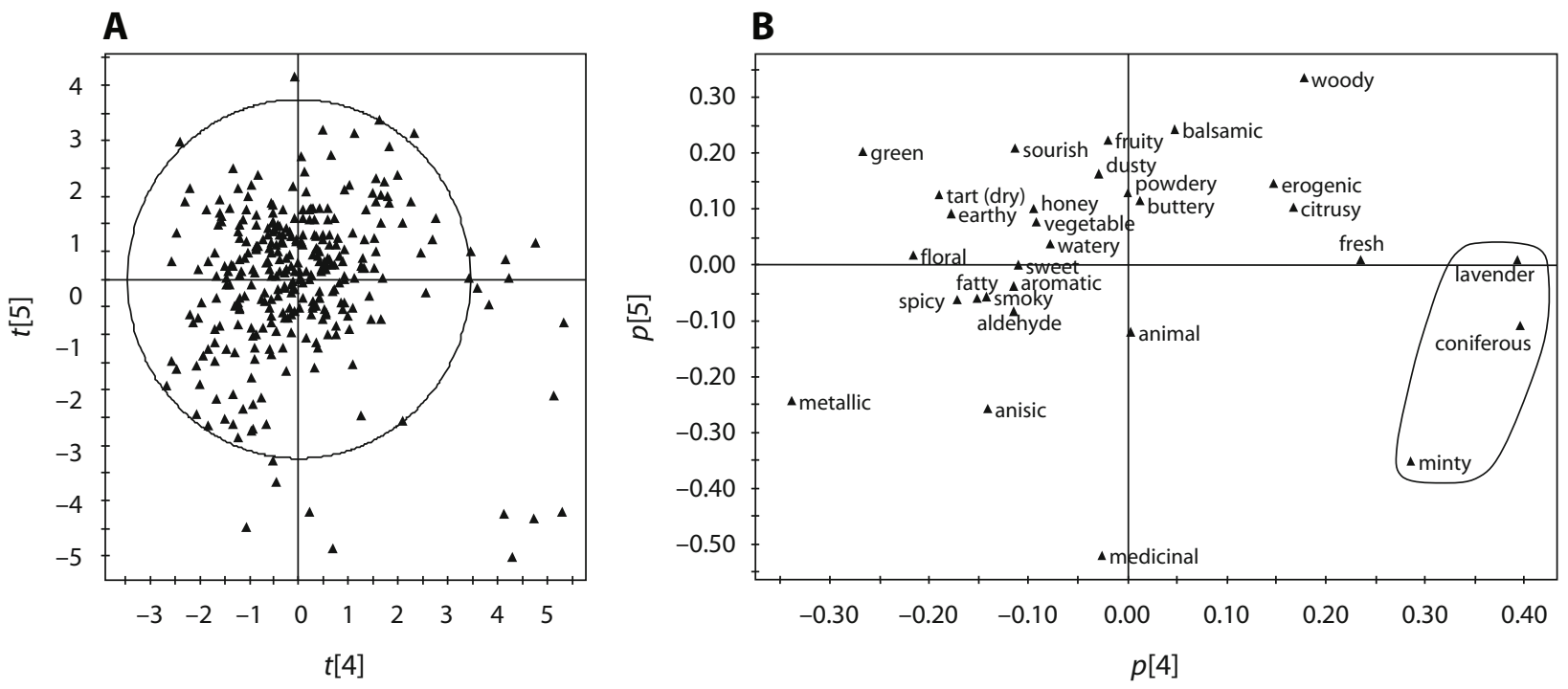

Figure 4. (A) Score plot ( $t[5]$ vs. $t[4])$ and (B) loading plot ( $p[5]$ vs. $p[4])$ corresponding to PC4/PC5 from the PCA conducted with the $B-H$ database. Variables were autoscaled prior to the analysis.

phenolic note that is present in the reference material for smoky (Table 1). Klein (1947) also regarded phenolic and burnt as related descriptors.

Minty, coniferous, and lavender present the highest loadings in PC4 (Figure 4) and appear close to each other in Figure 2. This common pattern is probably due to a camphoraceous note shared by the reference materials of these descriptors. Actually, according to Müller (1992), camphor-like notes are present in lavandin, rosemary, and conifer oils, among others. In a reported study of Arctander's database, minty, camphoraceous, and pine were grouped in the same cluster (Abe et al., 1990). Other works have also reported a similarity between camphor and minty (Chastrette et al., 1988), between camphoraceous and piney (Chastrette et al., 1991), and between camphoraceous and lavender (Calkin \& J. S. Jellinek, 1994).

Effect of the selected references on the similarities between descriptors. Brud (1986) asked 120 perfumers to indicate perfume raw materials that they would choose as representative for different odor descriptors. We compared the results of this survey with the references selected by Boelens and Haring (1981). Cedarwood oil and civet absolute were the preferred materials for woody and animal, respectively. The same criteria were used in the B-H database. Jasmine absolute was the second material most frequently chosen for floral, and eugenol was also the second choice for spicy. However, only $2 \%$ of respondents selected olibanum resinoid as a standard for balsamic, and the same percentage resulted in the case of methyl heptin carbonate as a reference for green. None of the perfumers who took the survey chose undecylenic alcohol or hexadecanal as a standard for fatty or fruity, respectively. Nonetheless, methyl heptin carbonate was one of the six consensus materials chosen by a group of experts as a reference for green, and hexadecanal was one of the standards proposed for fruity (Harper, 1975).
Galbanum resinoid and patchouli oil were the materials most frequently selected for green and earthy, respectively (Brud, 1986). By contrast, Boelens and Haring (1981) assigned these materials to tart (dry) and dusty, respectively (Table 1). Therefore, if they had been used in the original study according to the preferred selection of Brud's survey, green would appear instead of tart (dry) in the loading plots, and earthy would appear instead of dusty.

Metallic notes are reminiscent of the flavor caused by metal ions, such as iron or copper, in solution. Some perfumes also contain metallic nuances, and several studies have found a similarity between metallic and geranium (Abe et al., 1990; Chastrette et al., 1988). Bay oil was the reference material for metallic. It smells spicy-fresh (Table 1), and the metallic character is probably a minor note. This observation suggests that perfumers might select a material as a reference for a particular aspect, although this odor character is not the dominating note. Most likely, naive participants will not describe the odor of bay oil as "metallic," a term that they would apply to smells with a strong metallic character. This reason might explain why metallic was classified by Jeltema and Southwick (1986) as falling within the solvent cluster along with descriptors such as paint, chemical, and alcoholic.

Interpretation of PC2. Perfumes with floral accents are generally considered to be especially feminine (Müller, 1992). Actually, $60 \%$ of the feminine fragrances in the H\&R Fragrance Guide (Glöss, 1991) appear under the floral category, but only $8 \%$ of masculine fragrances are listed under a floral subcategory of fougère or citrus. J. S. Jellinek (1992) developed a two-dimensional mapping of commercial perfumes, and one of the dimensions was floral (romantic) versus not floral. Floral is one of the variables with highest $p[2]$, which might indicate that PC2 discriminates odor descriptors that are more characteristic of women's versus men's fragrances. This hypothesis, pro- 
Table 6

Frequency of Occurrence of Odor Character Descriptors Used by the H\&R Fragrance Guide (Glöss, 1991) to Describe the Top, Middle, and Base Note of 820 Commercial Perfumes

\begin{tabular}{|c|c|c|c|c|c|c|c|c|c|c|c|c|c|}
\hline \multicolumn{7}{|c|}{ Feminine Descriptors $(\% \mathrm{~F}>\% \mathrm{M})$} & \multicolumn{7}{|c|}{ Masculine Descriptors $(\% \mathrm{M}>\% \mathrm{~F})$} \\
\hline \multirow[b]{2}{*}{ Descriptor } & \multicolumn{2}{|c|}{ Top Note } & \multicolumn{2}{|c|}{ Middle Note } & \multicolumn{2}{|c|}{ Base Note } & \multirow[b]{2}{*}{ Descriptor } & \multicolumn{2}{|c|}{ Top Note } & \multicolumn{2}{|c|}{ Middle Note } & \multicolumn{2}{|c|}{ Base Note } \\
\hline & $\% \mathrm{~F}$ & $\% \mathrm{M}$ & $\% \mathrm{~F}$ & $\% \mathrm{M}$ & $\% \mathrm{~F}$ & $\% \mathrm{M}$ & & $\% \mathrm{~F}$ & $\% \mathrm{M}$ & $\% \mathrm{~F}$ & $\% \mathrm{M}$ & $\% \mathrm{~F}$ & $\% \mathrm{M}$ \\
\hline Fruity & 32.5 & 1.1 & 4.6 & 0.8 & 0.7 & 0.0 & Fresh & 52.1 & 92.6 & 2.9 & 13.6 & 0.2 & 1.9 \\
\hline Sweet & 0.0 & 0.0 & 10.2 & 1.1 & 23.8 & 13.4 & Herbaceous & 2.0 & 42.5 & 0.2 & 1.6 & 0.0 & 0.5 \\
\hline Green & 34.2 & 23.2 & 5.3 & 3.0 & 0.2 & 0.0 & Woody & 0.0 & 0.8 & 5.5 & 38.4 & 34.2 & 40.9 \\
\hline Elegant & 0.0 & 0.0 & 10.6 & 0.0 & 0.2 & 0.0 & Resinous & 0.2 & 0.0 & 0.9 & 13.4 & 0.0 & 1.6 \\
\hline Light & 0.7 & 0.0 & 3.3 & 0.0 & 0.0 & 0.0 & Dry & 3.1 & 1.4 & 2.9 & 13.6 & 0.0 & 0.0 \\
\hline Cool & 1.1 & 0.0 & 4.2 & 1.9 & 0.0 & 0.0 & Lavendaceous & 0.2 & 0.8 & 0.0 & 0.8 & 0.0 & 0.0 \\
\hline Mild & 0.0 & 0.0 & 0.2 & 0.0 & 4.2 & 1.4 & Tobacco & 0.0 & 0.0 & 0.0 & 0.3 & 0.0 & 0.5 \\
\hline Delicate & 0.2 & 0.0 & 2.4 & 0.0 & 0.0 & 0.0 & & & & & & & \\
\hline Sultry & 0.0 & 0.0 & 0.9 & 0.0 & 0.2 & 0.0 & & & & & & & \\
\hline
\end{tabular}

Note-A given descriptor is classified as feminine if the percentage of feminine fragrances with the top, middle, or base note labeled with that descriptor $(\% \mathrm{~F})$ is higher than the percentage in the case of masculine fragrances $(\% \mathrm{M})$.

posed by Zarzo (2008b) in a previous study of the B-H database, was further investigated here using the H\&R guide. It contains the semantic odor description of 820 commercial perfumes (367 men's and 453 women's) according to top, middle, and base notes. For each descriptor in the guide, we counted the number of times that it was applied to describe feminine fragrances. The relative frequency of occurrence was obtained by dividing these values by the total number of feminine fragrances. The same procedure was applied with masculine fragrances (Table 6). Some additional descriptors with a lower frequency of occurrence that are used in only one of the phases (top, middle, or base notes) are listed in Table 7.

Most of the 30 descriptors in the B-H database have a direct correspondence with the terms used by the H\&R guide. Perfumers use resinous to describe the odors of gums exuded from trees, particularly pines and other evergreens that present a somewhat harsh piney note from the turpentine oil usually present (Thiboud, 1991). The reference material for coniferous smells piney (Table 1), and hence it can be matched with resinous. Herbaceous refers to a fragrance that is natural, hay-like, and reminiscent of herbs that are used as food or medicine. Some examples are chamomile, lavender, rosemary, and sage (in particular, clary sage) (Müller, 1992). Taking into ac- count that clary sage oil was the reference for vegetable, this descriptor was considered equivalent to herbaceous. Smoky is not included in the H\&R guide, but it could be matched with leathery, given that smoky notes are used mainly in masculine perfumes to create natural leather effects (Müller, 1992). The similarity between smoky and leathery was also reported by Abe et al. (1990) (see Table 5).

As shown in Tables 6 and 7, the descriptors that were applied more often to feminine rather than masculine fragrances were classified as feminine. If the opposite applied, the attribute was classified as masculine. Despite the low frequency of occurrence of lavendaceous (Table 6), this descriptor was classified as masculine because lavender oil is used especially in masculine notes (Müller, 1992). Erogenic was not included in the H\&R guide, but it was regarded as feminine, because erogenous components are to be avoided in men's fragrances (J. S. Jellinek, 1997). Given the similarity between erogenic and animal (Table 2, Figure 3), and taking into account that the latter is seldom used in the H\&R guide, both descriptors were classified as feminine. This criterion is consistent with the classification of sensual and warm as feminine descriptors (Table 7). Actually, sensual refers to perfumes that have an erotically stimulating effect and that are usu-

Table 7

Frequency of Occurrence of Odor Character Descriptors Used by the H\&R Fragrance Guide (Glöss, 1991) That Are Only Applied to Describe the Top, Middle, or Base Note

\begin{tabular}{|c|c|c|c|c|c|c|c|c|}
\hline \multicolumn{6}{|c|}{ Feminine Descriptors $(\% \mathrm{~F}>\% \mathrm{M})$} & \multicolumn{3}{|c|}{ Masculine $(\% \mathrm{M}>\% \mathrm{~F})$} \\
\hline Descriptor $^{\mathrm{a}}$ & $\% \mathrm{~F}^{\mathrm{b}}$ & $\% \mathrm{M}^{\mathrm{c}}$ & Descriptor $^{\mathrm{a}}$ & $\% \mathrm{~F}^{\mathrm{b}}$ & $\% \mathrm{M}^{\mathrm{c}}$ & Descriptor $^{\mathrm{a}}$ & $\% \mathrm{~F}^{\mathrm{b}}$ & $\% \mathrm{M}^{\mathrm{c}}$ \\
\hline T_aldehydic & 29.4 & 1.9 & M_precious & 4.2 & 0.0 & B_mossy & 15.9 & 49.0 \\
\hline B_powdery & 53.2 & 36.8 & M_classic & 4.0 & 0.0 & T_citrusy & 3.5 & 11.2 \\
\hline B_sensual & 15.5 & 0.0 & M_narcotic & 0.9 & 0.0 & B_ambery & 13.7 & 20.4 \\
\hline B_warm & 21.6 & 10.6 & B_oriental & 0.7 & 0.0 & B_musky & 0.7 & 1.9 \\
\hline B_balsamic & 13.9 & 3.5 & M_honey & 0.2 & 0.0 & M_ozonic & 0.0 & 0.5 \\
\hline B_feminine & 6.2 & 0.0 & M_watery & 0.2 & 0.0 & M_algoid & 0.0 & 0.3 \\
\hline M_radiant & 4.2 & 0.0 & B_animalic & 0.4 & 0.3 & B_earthy & 0.0 & 0.3 \\
\hline
\end{tabular}

aThe prefix "T_," "M_," or "B_" indicates that the descriptor is only applied to top, middle, or base notes, respectively. An additional 15 descriptors with very low frequencies of occurrence are not listed. bPercentage of feminine fragrances labeled with a given descriptor. cPercentage of masculine fragrances labeled with a given descriptor. 


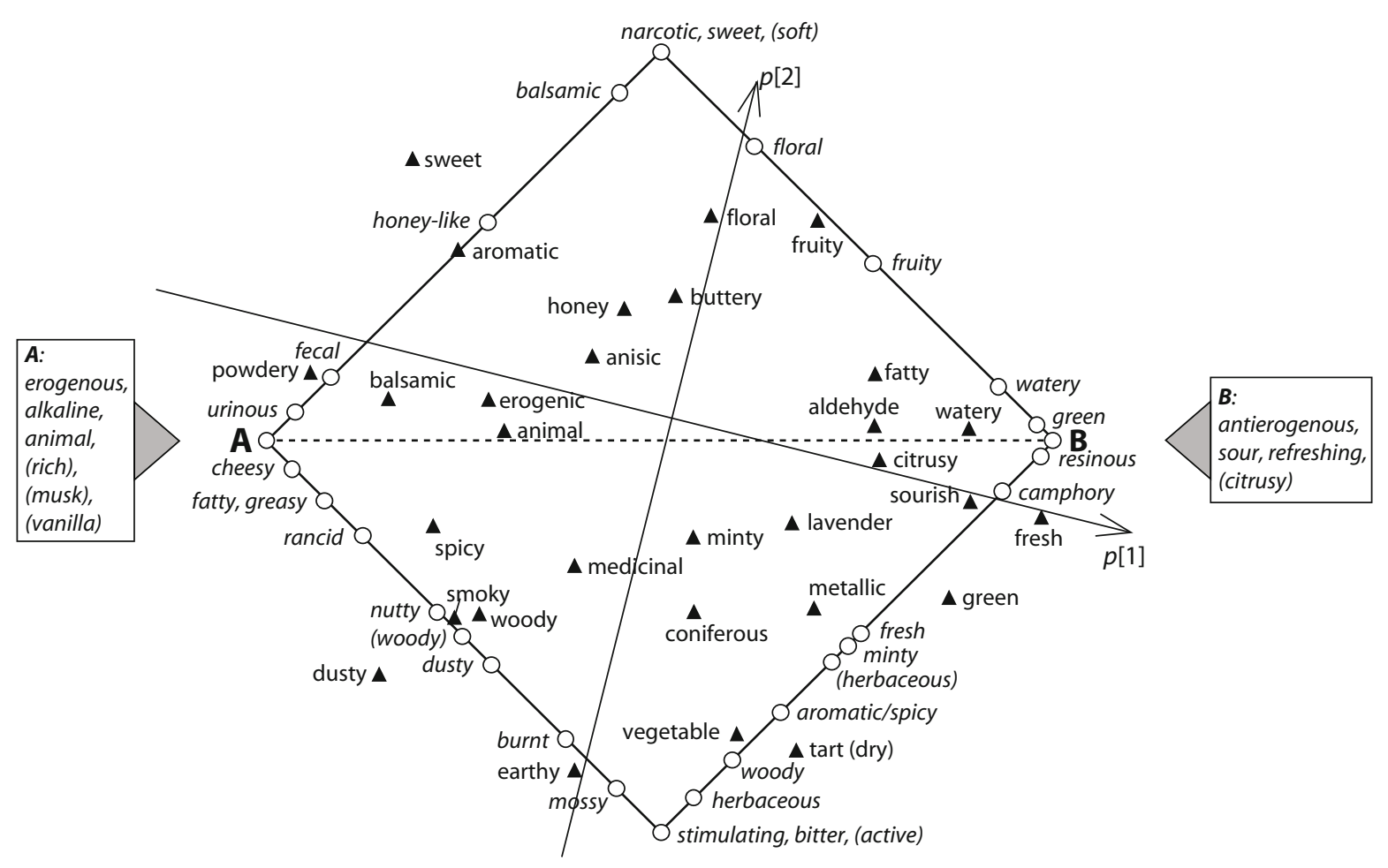

Figure 5. Rotated loading plot for PC1/PC2 from the PCA conducted with the B-H database. The dashed line corresponds to the dotted line indicated in Figure 2. The odor effects diagram (P. Jellinek, 1951, 1997) is also indicated for comparison purposes (odor descriptors in italics). Descriptors within parentheses correspond to the simplified diagram proposed by Calkin \& J. S. Jellinek (1994).

ally characterized by an accentuated portion of animalic components and exotic blossom notes (Müller, 1992). Warm perfumes also contain a high portion of animalic ingredients (Müller, 1992).

A dotted line was drawn in the PC1/PC2 plot (Figure 2). Interestingly, all descriptors classified as feminine appear above the line, and the opposite applies to masculine descriptors. The only exception is green, a general term used in perfumery for the odors reminiscent of freshly cut grass, leaves, stems, and so on (Müller, 1992). However, some authors distinguish green from grassy (Thiboud, 1991). The former refers to freshly cut leaves such as violet leaf absolute, which displays floral notes of violets. Grassy is used for a specific type of green note, freshly cut grass, which suggests dry accents (Thiboud, 1991). Taking into account that dry notes are used mainly in masculine perfumes (Müller, 1992), the position of green below the dotted line suggests that it can be interpreted as grassy.

Comparison of results with $P$. Jellinek's odor map. Figure 2 was rotated clockwise until the dotted line became horizontal. Jellinek's odor map was rotated $90^{\circ}$ clockwise, and, next, both plots were overlaid (Figure 5). The position of descriptors on the odor effects diagram (empty circles) corresponds to the original diagram as it was published in the first edition (P. Jellinek, 1951). A strikingly similar position is observed between descriptors in the $\mathrm{B}-\mathrm{H}$ database and the corresponding terms in Jellinek's odor map, except in some cases that are discussed in detail.
Burnt and smoky are semantically related descriptors, and they appear close to each other in Figure 5. Birch tar oil was the reference selected by P. Jellinek (1997) for burnt, and cade oil was assigned to smoky in the B-H database. Both materials smell tar-like, smoky, and phenolic (Brechbill, 2007). The proximity between lavender and camphory is also appealing because lavender scents present a camphoraceous note (Calkin \& J. S. Jellinek, 1994). Coniferous can be matched with resinous as mentioned above. The latter appears very close to refreshing, but, according to our results, it seems more appropriate to place resinous closer to coniferous.

Earthy notes give the impression of such things as freshly turned earth, forest soil, mold, moss, must, roots, yeast, and mushrooms. In perfumery, earthy essences are derived from a variety of mosses such as oakmoss, treemoss, and lichens. Oakmoss was the selected reference for earthy in the B-H database and, hence, it can be matched with mossy. The similarity of both descriptors is reflected in Figure 5.

Nutty was included in a later version of the odor effects diagram (see J. S. Jellinek, 1992). This descriptor is not far from woody, spicy, and earthy in Figure 5. Consistent with this result, Klein (1947) grouped nut-like and woody odors within the same cluster, whereas Abe et al. (1990) classified walnut (a nutty odor) as earthy. Moreover, nutty descriptors appeared close to spicy, cinnamon, and earthy in a reported analysis of semantic odor profiles (Zarzo \& Stanton, 2006). 
If the correspondence between vegetable and herbaceous is taken into account, the proximity of both descriptors is intuitively appealing. The position of green in the $\mathrm{PC} 1 / \mathrm{PC} 2$ plot is consistent with the relative position of this descriptor in the odor map reported by Thiboud (1991). Phenyl acetaldehyde dimethyl acetal was regarded by P. Jellinek (1997) as a reference for green. This odorant is contained in the $\mathrm{B}-\mathrm{H}$ database, and the floral score is 6 (on a $0-9$ scale). By contrast, methyl heptin carbonate (the reference selected in the $\mathrm{B}-\mathrm{H}$ database) was given a floral score of 3 . The different floral character of both references would explain why the position of green in the PC1/PC2 plot is not coincident with P. Jellinek's criterion.

Peru balsam is often regarded as a standard for balsamic (Brud, 1986), and this criterion was adopted by P. Jellinek (1997). This material smells balsamic, sweet, and reminiscent of vanilla (Brechbill, 2007; Rimmel, 1895). Olibanum resinoid, the reference selected by Boelens and Haring (1981), does not smell so sweet (Table 1), and it is probably for this reason that P. Jellinek (1997) located balsamic close to the sweet corner of the odor effects diagram, but it appears more distant in the PC1/PC2 plot.

Eugenol was chosen by Boelens and Haring (1981) as a standard for spicy, whereas safrole was assigned to aromatic/spicy (P. Jellinek, 1951). Zwaardemaker (1925) classified eugenol as aromatic/spicy, but safrole was regarded as aromatic/aniseed. Thus, aromatic/spicy in Figure 5 should be matched with anisic instead of with spicy. This interpretation would explain the discordant position between aromatic/spicy and spicy. Although spices and some herbs are used as culinary condiments, spicy odors are basically perceived as warming or hot in character, as opposed to the neutral or cool effects of herbal notes. Taking into account that PC1 can be interpreted as a warm/ cool dimension, as discussed below, the position of spicy derived from the $\mathrm{B}-\mathrm{H}$ database seems appropriate.

Eugenol and safrole are contained in the B-H database, and their odor profile is significantly different. In the case of safrole, the highest scores assigned by the panel correspond to anisic (7), vegetable (5), and spicy (4). The vegetable character of safrole supports the position of aromatic/spicy close to herbaceous in the odor effects diagram. Interestingly, Aftelier (2006) classified fennel oil (the reference for anisic) as herbal-anisic. But this similarity is not reflected in the B-H database, because the correlation between anisic and vegetable is very low $(r=.12)$. Anisic appears close to the center of the PC1/ PC2 plot because it yields a low correlation with the rest of the variables. It is unclear where this descriptor fits best on the odor effects diagram.

The position of fatty is also discordant, probably because of the different odor character of the reference materials. Fatty, greasy appears near the erogenous corner of the diagram because nonyl alcohol (the reference of this descriptor assigned by P. Jellinek, 1997) was regarded as erogenous in a sensory experiment that assessed the erotic power of about 200 perfume materials (P. Jellinek, 1997). This similarity between fatty and erogenous was also reported by different authors. According to Müller (1992), fatty notes are reminiscent of the smell of human skin in small doses, and hence they can contribute to the erotic effect of a perfume. Jeltema and Southwick (1986) classified oily, fatty as animal-foul (Table 5). Fatty and rancid are related descriptors, as shown in the diagram, because rancid odors are caused by the oxidation of fats.

Undecylenic alcohol was the selected reference for fatty in the $\mathrm{B}-\mathrm{H}$ database. The position of this descriptor in the loading plot reveals that this odorant smells fresh, which is actually the case (Table 1). Interestingly, P. Jellinek (1997) regarded this odorant as antierogenous. Brud (1986) reported that 1-decanol and 1-dodecanol were the odorants most frequently chosen among perfumers as standards for fatty. The latter was perceived as erogenous, and the former was somewhat intermediate of erogenous and antierogenous (P. Jellinek, 1997). Therefore, the position of fatty in the odor effects diagram strongly depends on the selected reference. This observation highlights the need to achieve a consensus in assigning reference materials to odor descriptors as a prerequisite for developing standard maps of odor descriptors.

The narcotic dimension of odor effect: A discussion. According to P. Jellinek (1997), narcotic odorous materials have certain female characteristics, whereas the stimulating materials present certain male characteristics. Thus, the underlying dimension in odor descriptor space based on the polarity feminine/masculine that was found in our study is consistent with the narcotic/stimulating dimension of odor effects. Similarly, Thiboud (1991) obtained a two-dimensional odor map, and the pair of contrasting descriptors men-husbands versus young wivesmothers was the polarity that better explained one of the dimensions. Describing scents as feminine versus masculine is probably more intuitive than is narcotic versus stimulating, given that the narcotic or stimulating effect of the individual perfume materials has yet to be proven scientifically. Narcotic is used in perfumery to describe the sweet, mellow, floral-balsamic fragrance of certain flowers said to be intoxicating (e.g., narcissus, tuberose, or ylang ylang). Consistent with this criterion, the position of narcotic on the odor effects diagram is somewhat intermediate of floral and balsamic.

P. Jellinek (1997) associated sweet and bitter with narcotic and stimulating effects, respectively. Sweet is a term common to tastes and flavors that has two applications in perfumery: (1) It can describe a rich fragrance with the ambrosial characteristics of a sweet taste (e.g., balsamic scents). (2) It can also refer to the perfumer's changes in a fragrance that make the odor smoother or softer when it is too dry, leafy, or bitter (Thiboud, 1991). Taking into account that smooth, soft, and feminine are similar descriptors, as described below, the second meaning supports the correspondence between sweet and narcotic proposed by P. Jellinek (1997). However, the analysis of the B-H database suggests that sweet is better mapped somewhere between the position of narcotic and erogenous. A similar result was observed in the odor map reported by Thiboud. Boelens and Haring (1981) assigned sweet to heliotropin, which smells very warm and floral-narcotic (Table 1). As- 
suming that warm and erogenous are related descriptors, as discussed below, it is intuitively appealing that the position of sweet in the PC1/PC2 plot is somewhat intermediate between erogenous and narcotic (Figure 5).

Bitter is one of the four basic taste sensations identified by human taste buds. Although perfumers do not largely agree on what is or what is not a bitter smell (Thiboud, 1991), this attribute is often applied to perfume odors that have a metallic green quality, without sweetness. Consistent with this criterion, P. Jellinek (1997) regarded bitter and sweet as opposite descriptors. But the polarity sweet/ bitter is probably better interpreted as sweet/dry, because $d r y$ usually refers to the absence of sweetness in a fragrance (Aftel, 2001; Thiboud, 1991). The descriptor most dissimilar to sweet in the B-H database is tart (dry), and both variables appear at opposite positions in the PC1/ PC2 plot. P. Jellinek (1997) postulated that sweet/bitter could be matched with the narcotic/stimulating dimension. But the analysis of the B-H database does not support this hypothesis, because the sweet/dry polarity clearly departs from the vertical axis in Figure 5.

The associations sweet-feminine and dry-masculine are well known in perfumery. Masculine fragrances are generally less floral than feminine perfumes and contain dry notes of leather, tobacco, herbs, spices, mosses, and woods. Bitter accents are also characteristic of men's fragrances (Müller, 1992). Conversely, most feminine fragrances smell floral, and this descriptor yields the highest correlation with sweet in the B-H database (Table 3). Nonetheless, floral and sweet account for independent dimensions of odor character. Actually, the reference selected by P. Jellinek (1997) for floral (hydroxycitronellal) is contained in the B-H database, and the sweet score of this odorant is rather low ( 2 on a $0-9$ scale).

The sweet smell of certain odors seems to be caused by associative learning due to the co-occurrence of such odors and tastes (Stevenson, Prescott, \& Boakes, 1995). A similar interpretation applies for perfumes. Kirk-Smith and Booth (1987) suggested that a fragrant scent can acquire subtle meaning through learned associations if it is experienced in a meaningful situation. The perceived odor may evoke recognition of the source as well as any thing, place, persons, or effect that might be associated with it. The same reasoning would suggest that the relationship between sweet and feminine odors might be the result of a stable cross-modal association developed in the first months of our life between the taste of food while breastfeeding (milk clearly tastes sweet) and the odor of the food source (feminine smell). This hypothesis is mere speculation, but it deserves to be investigated further.

Interpretation of PC1. Fresh was long ago proposed as an independent category of odors (Bain, 1855). Similarly, it is one of the five standard families of perfumes proposed by Edwards (2006), which is subdivided into three categories: citrus, green, and water. Different authors suggest that fresh, in relation to the effect of a fragrance, is a perception not easy to define (Calkin \& J. S. Jellinek, 1994; Müller, 1992; Thiboud, 1991). Some experts maintain that fresh is an effect introduced into a fragrance by the use of citrus oils or certain aldehydes. Other perfumers use fresh when referring to the green note of recently cut leaves. A fresh fragrance is usually considered invigorating, nature inspired, or reminiscent of the outdoors and is typified by green, citrus notes (Thiboud, 1991). Interestingly, fresh is the descriptor with the highest loading in PC1, and it is located close to watery, citrusy, aldehydic, and green in the PC1/PC2 plot (Figure 2). Thus, PC1 can be interpreted as a dimension of freshness. In order to better understand the psychological aspects associated with this dimension, it is of interest to discuss our results in relation to the work of P. Jellinek (1997).

Interpretation of $\mathrm{PCl}$ as erogenous versus antierogenous. The position of fresh and related descriptors in the PC1/PC2 plot has a direct correspondence with the odor effects diagram (Figure 5). Hence, PC1 could be interpreted as the erogenous/antierogenous dimension of odor effects. P. Jellinek (1997) regarded refreshing and fresh as different odor descriptors. The former was associated with antierogenous, sour, and citrusy, as well as with the perfume type Eau de Cologne, with the citrus note emphasized. By contrast, fresh was basically associated with green-herbaceous odors, which were supposed to produce antierogenous and stimulating effects. Methyl heptin carbonate (the selected reference for green in the B-H database) was classified as fresh (i.e., refreshing-stimulating) by P. Jellinek (1997). The position of green in the loading plot, somewhat intermediate of antierogenous and stimulating (Figure 5), is consistent with this criterion. Masculine fragrances often contain a high percentage of fresh ingredients (Müller, 1992). The classification of fresh and citrusy as masculine (Table 6) is consistent with the position of both terms below the dashed line in Figure 5 .

The association between sour and refreshing proposed by P. Jellinek (1997) was also reported by a sensory study conducted with 50 participants who were asked to rate 11 odorant materials on a numeric scale according to 30 sense-descriptive adjectives. A factor analysis of the resulting data led to three relevant factors. One of them, interpreted as clarity, was basically determined by the terms cool, clear, and sour (Higuchi, Shoji, \& Hatayama, 2004). Taking into account that clarity refers to fresh scents (Tisserand, 1988), this factor corresponds to the dimension of freshness.

The correspondence between sour and citrusy (Calkin \& J. S. Jellinek, 1994) is based on a cross-modal association between taste and smell. Lemon tastes sour and, hence, lemon oil (a characteristic citrus scent) was regarded as a reference for sour (P. Jellinek, 1997). In the context of perfumery, fruity refers to the sweet-sour odors of natural fruits excluding citrus. Consistent with this description, the position of fruity in Figure 5 is intermediate between sweet and sour. Excesses of fruitiness are to be avoided in perfumes, because they would suggest food flavors rather than cosmetic products (Müller, 1992).

P. Jellinek (1997) probably located alkaline and sour at opposite corners of the diagram because they are semantically opposite terms. But the similarity alkaline-erogenous is arguable. By contrast, the association animal-erogenous 
(Figure 5) seems more appropriate because animalic materials (i.e., natural or synthetic odorants smelling like extracts from animal origin) are often used in perfumes to add an erotic tone (P. Jellinek, 1997; Moran, 2000; Müller, 1992). The best-known products of the animal kingdom in the perfume industry are civet, musk, castoreum, and ambergris. They often smell unpleasant, obtrusive, and fecal in concentrated form. Correctly diluted, however, they are an irreplaceable ingredient in many perfumes, providing warmth and a sensual feel (Müller, 1992). It is therefore not surprising to find fecal close to the erogenous corner of the odor effects diagram (Figure 5). Similarly, Jeltema and Southwick (1986) classified fecal, urine, and sweaty in a cluster referred to as animal-foul (Table 5).

Assuming that erogenous/antierogenous is a salient dimension of odor descriptor space, and taking into account the observed similarity between animal and erogenic $(r=$ 46), both descriptors were expected to be the ones most dissimilar to fresh (i.e., those located at the opposite position in the PC1/PC2 plot) among the 30 descriptors of the $\mathrm{B}-\mathrm{H}$ database. But this is not the case (Figure 2), given that animal and erogenic define an independent dimension, somewhat intermediate to PC3 and PC6 (Figure 3), which is orthogonal to PC1 (i.e., the dimension of freshness). Nonetheless, powdery is the descriptor most dissimilar to fresh (Table 3), and it presents a similarity with erogenic $(r=.49)$ because of certain erogenous and musky notes of the reference material (Table 1). Chastrette et al. (1991) grouped powdery, musky, and animal in the same cluster. The polarity erogenous/refreshing does not provide a good enough interpretation for $\mathrm{PC} 1$, and other complementary interpretations are discussed here.

Calkin and J. S. Jellinek (1994) also realized that animal is probably not the descriptor most dissimilar to fresh, and these authors proposed the term rich. The same criterion is applied by Edwards (2008), who considers fresh and rich to be opposite descriptors of fragrances, and who classifies as rich those fragrances in the family that produce the deeper impression. Rich and deep are similar terms in perfumery (Thiboud, 1991).

According to P. Jellinek (1997), women emphasize their feminine gender least when at work and most in their social life. This hypothesis explains why women prefer fresh fragrances (i.e., antierogenous) for informal daytime wear, whereas sensual perfumes are preferred for evening wear. This dimension of daytime versus evening in fragrance preference was reflected in a reported study conducted with 10 commercial perfumes that were rated according to different attributes (J. S. Jellinek, 1992). The preference of antierogenous scents for daytime is also reflected in the two-dimensional odor map reported by Thiboud (1991), showing that morning and fresh are mapped close to each other, whereas evening and night appear at opposite positions of this odor map.

Interpretation of PC1 as heavy versus light. Light odors correspond to materials that have low boiling points and that tend to disperse quickly into the air because of their high volatility. The opposite applies to heavy odors that are often described as rich. The parameter that measures the lasting property of an odorant is called substantivity, and it is partly dependent on the degree of volatility. Perfume is a complex mixture of substances of different volatilities. Heavy perfumes are those in which the least volatile ingredients, such as mosses and animal scents, dominate (Thiboud, 1991). Conversely, light fragrances are those perceived as nonsweet with a fresh note that is predominant and that is often associated with citrus, greens, aldehydes, and delicate floral notes (Green, 1999; Moran, 2000). According to Tables 6 and 7, fresh, green, and aldehydic are frequently encountered at the top note of a fragrance (i.e., light odors), and these descriptors appear in Figure 2 with $p[1]>0$. Conversely, powdery, sweet, and balsamic generally correspond to base notes (i.e., heavy odors), and they are characterized by $p[1]<0$. Therefore, $\mathrm{PC} 1$ could also be interpreted as a heavy/light dimension. Given the association fresh-light-volatile, Edwards (2008) defines fresh as the most effervescent fragrances.

Different studies have also reported that heavy/light is a salient dimension in the perceptual space of cosmetic scents. A sensory study conducted with 90 German women (J. S. Jellinek, 1980) revealed that the dimension heavy/ light discriminated most strongly among the six perfumes used in that study. J. S. Jellinek (1992) developed a twodimensional map of fragrances based on the dimensions heavy/light and floral/nonfloral, which are not difficult concepts for consumers to understand. With the help of a pilot study, this author found that the scale warm/cool was readily understood by German female perfume users. Taking into account that these descriptors are more neutral, in terms of value judgment and of the relationship to odor intensity, J. S. Jellinek (1992) used warm/cool in place of heavy/light in the map of fragrances. This criterion seems appropriate, because heavy has many meanings in perfumery, and it is sometimes applied to describe intense odors (Thiboud, 1991). Moreover, warm and cool are semantically opposite concepts.

Interpretation of PCl as warm versus cool. Warm perfumes are often described as rich and deep (Thiboud, 1991), and they have a high proportion of animalic ingredients (Müller, 1992). Harper (1975) selected costus and amber (i.e., two animalic materials) as standards for warm. Hence, warm and erogenous are related descriptors. The reference materials for powdery, sweet, and balsamic in the B-H database are described as warm (Table 1). These are the attributes with the most negative loadings in PC1, whereas fresh is the descriptor with highest $p$ [1] (Figure 2). Thus, PC1 can also be regarded as a warm/ fresh dimension. A similar interpretation is deduced from a reported analysis of the language of French perfume advertising. It was found that warm and sensual were closely related descriptors, and the dimension warm/fresh was the most important axis of the semantic field of fragrances (Blumenthal, 1979). Another study shows the classification of 140 commercial perfumes according to the warm/ cool dimension (J. S. Jellinek, 1990).

Fresh, cool, and warm are terms semantically associated with temperature. On one hand, a temperature similar to that of our body tends to be described as warm. On the other hand, the materials whose smell is reminiscent of the human body odor are erogenous (J. S. Jellinek, 1997). 
This association might explain the similarity between warm and erogenous. Klein (1947) also suggests that the human flesh aura evokes animalic scents.

J. S. Jellinek (1992) reports the results of a sensory study conducted with 10 commercial perfumes that were rated according to different attributes. The dominant factor distinguished fresh fragrances, which were described as "summer," from sensual and passionate fragrances, which were associated with winter. These results are consistent with the hypothesis that fresh evokes a cooling temperature and hence fresh perfumes are preferred in summer. Conversely, sensual (i.e., erogenous) perfumes evoke warming temperatures and are good for cooler seasons. Oriental perfumes are reminiscent of fragrances from the Orient and contain sensual ingredients, often heavy blends of oriental balsamic resins, opulent flowers, sweet vanilla, and musks (Edwards, 2008). These ingredients evoke warmth, and hence oriental fragrances are mostly used as "winter" perfumes (Müller, 1992).

A cool scent often suggests a clean, fresh, outdoor quality, possibly with green, citrus-mint undertones (Thiboud, 1991). Harper (1975) selected menthol and camphor as references for cool, cooling. These odorants share a mintyfresh odor character. Minty odors produce a trigeminal effect that is perceived as cooling, which would explain the similarity between fresh and minty $(r=.32)$. In the Dravnieks database, minty yields the highest correlation with cool, cooling $(r=.82)$. This similarity suggests that cool should be preferentially applied to the perception of freshness associated with a trigeminal effect. Therefore, we suggest that $\mathrm{PC} 1$ is better interpreted as warm/ fresh instead of warm/cool. Trigeminal nerve receptors are responsible for tactile, pressure, pain, and temperature sensations in the nasal cavity. A number of chemical trigeminal stimulants produce effects described as irritating, tingling, pungent, cooling (menthol), or hot (capsaicin). The interaction between the trigeminal and olfactory systems is an important determinant of odor sensations (Hummel \& Livermore, 2002).

Interpretation of $\mathrm{PCl}$ as watery versus powdery. The observed similarity between fresh and watery $(r=.37)$ is intuitively appealing because water is refreshing. Moreover, fruits (citrus or noncitrus), vegetables, and plants are natural products with a high water content, and their respective odor descriptors appear in Figure 2 with $p[1]>0$. Conversely, spicy, woody, dusty, and smoky evoke products with a low water content, and these descriptors present $p[1]<0$. Therefore, $\mathrm{PC} 1$ could also be regarded as an underlying dimension that evokes presence versus absence of water. This interpretation is not surprising, given that water is so abundant in nature.

Powdery is the descriptor most dissimilar to fresh (Table 3), and it evokes lack of water. The term powdery is preferable to $d r y$, because the latter is usually applied to describe the lack of sweetness in perfumery odors (Aftel, 2001; Thiboud, 1991). In the Dravnieks database, drypowdery is a single descriptor, and it yields the highest correlation with woody and musk. Müller (1992) defines powdery as the fragrance effect produced by the interaction of long-lasting (i.e., heavy) mossy, woody, sweet ele- ments. The position of powdery, somewhat intermediate among earthy, woody, and sweet in Figure 5, supports this definition. Moreover, powdery yields the highest correlation with erogenic, dusty, sweet, balsamic, and woody. A mixture of musk ketone and coumarin (a sweet-herbaceous odorant) was selected as a reference for powdery in the B-H database (Table 1), and Harper (1975) also selected both materials as standards for powdery. Consistent with this criterion, powdery is often defined as a sweet-dry, somewhat musky odor.

Brechbill (2007) classifies odorant materials into 19 categories, one of which is fresh air, ozone. Fresh is sometimes applied to the clean and invigorating scent of early morning air or perhaps an ozonic smell (Thiboud, 1991). This definition fits the description of the water family of fragrances proposed by Edwards (2008), which is characterized by marine and aquatic notes. According to this author, water notes capture the pure scent of a waterfall, the ozonic aroma of wet air after a thunderstorm, and the cool freshness of sea air; and they evoke the scent of soft sea breezes. Green and citrus also refer to fresh scents, and, interestingly, lemon odors associated with green notes may evoke sea (Thiboud, 1991). Thus, although water is odorless, watery is used to describe the scent of natural environments associated with water. Taking into account the alternation of seasons, some being drier than others, the ability of olfaction to recognize water-related seasonal odors from the environment may be related to chronobiological annual rhythms.

In summary, $\mathrm{PC} 1$ can be interpreted as a dimension of freshness that has a direct correspondence with the erogenous/antierogenous dimension of odor effect as well as other contrasting polarities such as rich/fresh, heavy/ light, warm/cool, and powdery/watery. Further research is encouraged to provide a better understanding of the psychological aspects involved in this underlying dimension.

\section{PCA of Thiboud's Database}

A variety of the studies mentioned above have reported that, although the hedonic dimension is usually the most salient in the multivariate analysis of numeric odor profile databases, that dimension rarely shows up in the analysis of semantic odor databases. Thus, it might be argued that the latter are more suitable for studying the underlying dimensions of odor descriptor space. Thiboud's (1991) database contains useful information to further investigate this issue. It comprises 119 materials selected as representatives of the main odor classes in perfumery: fresh, fruity, rosy, floral, balsamic, spicy, woody, agrestic, green, and citrus. Hence, the analysis of this database is supposed to reflect the similarities and dissimilarities among these categories of fragrances.

We analyzed the dichotomic matrix with PCA using the autoscaling pretreatment. In this case, there are 21 PCs with an eigenvalue $>1$. This number is obviously too high, and hence another criterion is necessary to determine how many components provide the relevant information. Table 4 shows the $Q^{2}$ value up to PC10. PC1 and $\mathrm{PC} 2$ are the only components that satisfy the crossvalidation criterion (i.e., $Q^{2}>Q^{2}$ limit). Thus, the PC1/ 


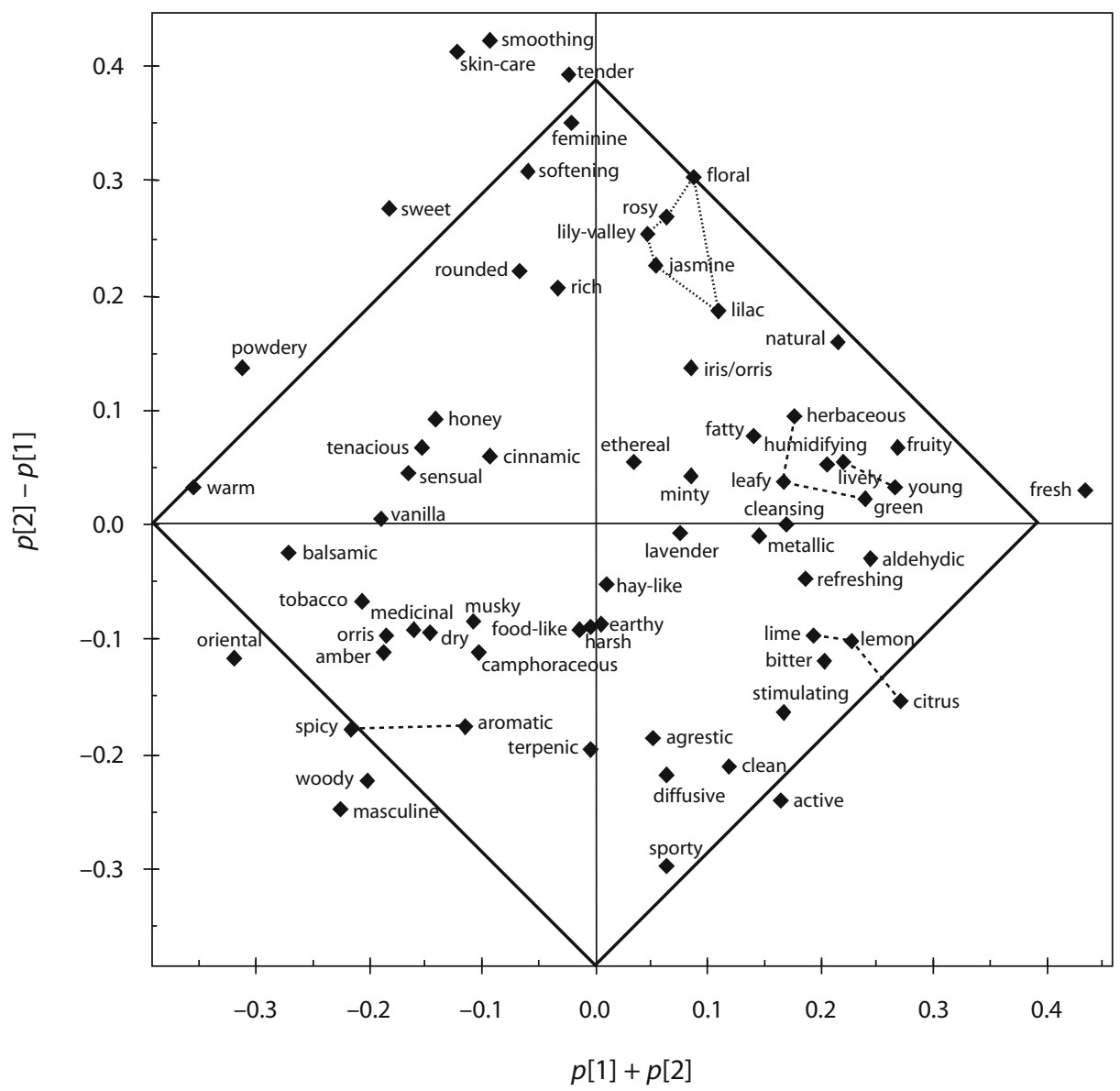

Figure 6. Rotated loading plot resulting from a PCA conducted with a dichotomic matrix of 66 odor descriptors. This matrix was obtained by coding numerically the semantic odor description of 119 perfume materials (Thiboud, 1991). Data were autoscaled prior to the PCA. In order to ease the comparison with Figure $5, p[2]-p[1]$ is plotted against $p[1]+p[2]$, which corresponds to a $45^{\circ}$ rotation of the loading plot for PC1/PC2. The square is intended to allow an easier comparison of this odor map with the position of descriptors in Jellinek's odor map (Figure 5). The dotted cluster groups floral descriptors. Dashed lines highlight similarities reflected by further components.

PC2 plot is expected to be a meaningful map of odor descriptors. Nonetheless, we also checked additional loading plots with different combinations of components up to PC5 and compared them with the PC1/PC2 plot in order to identify robust similarities among descriptors. PC1 and PC2 explain a similar amount of the data variance, given that their $R^{2} \mathrm{X}$ and $Q^{2}$ values are similar. In such cases, the underlying dimensions are sometimes better interpreted by conveniently rotating the $\mathrm{PC} 1 / \mathrm{PC} 2$ plot. We noticed that this plot was strikingly similar to Figure 5 after a $45^{\circ}$ clockwise rotation. This rotation can be achieved by plotting $p[2]-p[1]$ versus $p[1]+p[2]$ (Figure 6).

We applied the same procedure to the trichotomic matrix: A PCA was conducted after the autoscaling pretreatment, and, next, the PC1/PC2 plot was rotated. The resulting odor map (figure not shown) was very similar to Figure 6 . We found that the rotated loading plot obtained from the dichotomic matrix better resembled the two- dimensional map reported by Thiboud (1991); therefore, this plot is discussed in detail next.

Interpretation of the underlying dimensions according to the odor effects diagram. Sensual and tenacious (Figure 6) match approximately the position of erogenic in Figure 5. Taking into account that tenacious refers to odors with high substantivity (i.e., heavy), this result supports the interpretation of the $x$-axis as erogenous/ antierogenous or heavy/light. The polarity powdery/ watery is also reflected in Figure 6, because powdery and humidifying are located at opposite positions. Nevertheless, the warm/fresh interpretation seems to be the most appropriate given that fresh is the term with highest coordinate along the $x$-axis and, conversely, warm is located at the opposite extreme. Rich was expected to appear closer to warm and sensual, given the similarity among these descriptors mentioned above. However, rich is a rather subjective descriptor, and the results suggest that it was probably applied with a different meaning. 
Young and lively can be regarded as similar descriptors, because they appear close to each other in Figure 6; moreover, both attributes have the highest loadings in PC3. According to P. Jellinek (1997), 20-year-old people prefer fresh scents. A similar criterion can be deduced from the plot on the basis of the position of young.

Feminine and masculine appear as opposite variables. Hence, the vertical axis of Figure 6 can clearly be interpreted as femininity versus masculinity, which corresponds to the narcotic/stimulating dimension of odor effects. Calkin and J. S. Jellinek (1994) associated this dimension with the polarity soft versus active. Interestingly, softening is mapped next to feminine; furthermore, active appears at the opposite extreme of the plot. It is intuitively appealing that sporty and active are next to each other, because sports involve physical activity.

The rose odor reflects softness, femininity, and sensitiveness (Thiboud, 1991). This odor quality of rosy is also reflected in Figure 6, because it appears near softening and feminine. Smoothing and softening have a similar meaning, and consequently they are plotted close together. Skin-care is mapped close to feminine, probably because of a psychological association given that women use skin-care products much more often than do men. The feminine characteristic of tender is rather obvious, because this adjective is applied primarily to women. In the context of perfumery, rounded and harsh are opposite concepts. The former suggests balance, smoothness, and harmony (Thiboud, 1991). Interestingly, the same relationships among rounded, smoothing, and harsh can be deduced from the plot as well.

The observed similarity between soft and sweet was also reported in a sensory study using 11 odorants that were assessed according to 30 adjectives. Three relevant factors were identified. One of them, interpreted as softness, was basically determined by mild, soft, and sweet (Higuchi et al., 2004). Given the association softfeminine-sweet (Figure 6), and taking into account that mild is a feminine descriptor (Table 6), the factor of softness would correspond to the narcotic/stimulating dimension of odor effects.

Bitter usually refers to a dry perfume odor with a metallic green quality. Interestingly, the position of bitter in Figure 6 is similar to the position in Figure 5 of metallic and green, which are related descriptors (Table 5). The polarity sweet/bitter in Figure 6 does not match the odor effects diagram, but it corresponds approximately to sweet/ tart (dry) in Figure 5.

Interpretation of results according to the odor classification of Zwaardemaker (1925). One of the early odor classification systems is based on nine categories: ethereal, aromatic, fragrant (i.e., floral and balsamic), ambrosial, alliaceous, empyreumatic, caprylic, repulsive, and fetid (Zwaardemaker, 1925). The hypothesis that ethereal is a rather independent category is also reflected in Figure 6, because this descriptor is the one closest to the center. A similar result was obtained by Zarzo and Stanton (2006), who found a similarity between ethereal and chemical.

Aromatic yields the highest correlation with fragrant in the Dravnieks database, but Zwaardemaker (1925) considered both terms as independent categories of odors. Regarding aromatic odors, this author proposed five subcategories: camphoraceous, spicy, aniseed, citrus, and almond. Interestingly, camphoraceous and spicy are close to aromatic in Figure 6, but the loading plot for PC4/PC5 (figure not shown) suggests that aromatic was applied in Thiboud's (1991) database primarily to describe spicy odors. The position of spicy in Figure 6 is nearly the same as in the $\mathrm{PC} 1 / \mathrm{PC} 2$ plot from the $\mathrm{B}-\mathrm{H}$ database (Figure 5). We suggest that the same criterion could be adopted in future versions of the odor effects diagram.

Benzaldehyde was selected as a standard for aromatic by Harper (1975), but Zwaardemaker (1925) classified it as aromatic-almond. Edwards (2008) associates aromatic with fougère, which refers to the combination of fresh herbaceous lavender notes on a mossy foundation (Müller, 1992). This criterion is not consistent with the subcategories of aromatic odors proposed by Zwaardemaker. The term aromatic was used in former times to describe the fragrance impression of sweet balsams (Müller, 1992). This criterion was probably adopted by Boelens and Haring (1981), because they selected vanillin as a reference for aromatic. By contrast, Zwaardemaker regarded vanillin as a balsamic odor. Given the subjectivity of aromatic, which is not always applied in perfumery with the same meaning, it seems convenient to disregard it in future studies of odor profiling.

Ambergris exhibits notes of woody, dry balsamic, and tobacco-like (Table 1). This odor description is consistent with the position of amber in Figure 6. Zwaardemaker (1925) classified musk and amber odors (i.e., those resembling ambergris) as ambrosial. Interestingly, musky, amber, and dry are close to each other, and these descriptors are perceived as primarily masculine (see Table 7).

Further discussion of the resulting map of odor descriptors. Figure 6 suggests that balsamic, vanilla, honey, and cinnamic are similar descriptors. Interestingly, these descriptors are classified as balsamic in the Sigma-Aldrich (2003) catalog. The position of balsamic is consistent in the two odor databases analyzed (Figures 5 and 6), but this descriptor appears on the odor effects diagram closer to sweet. This result further suggests that the position of some descriptors in Jellinek's odor map should be revised.

The positions of fatty and aldehydic are consistent with the $\mathrm{PC} 1 / \mathrm{PC} 2$ plot from the $\mathrm{B}-\mathrm{H}$ database. Citrus, lemon, and lime are close to each other in Figure 6 as well as in the loading plot for PC3/PC4 (not shown). This relationship is obvious, because limes and lemons are citrus fruits. The proximity between citrus and stimulating suggests a masculine character of citrus odors that is also reflected in Table 7.

Orris and iris/orris are located at opposite positions, although they are semantically related. Orris is a perfume material extracted from the rhizomes of the iris plant (Iris pallida and other species) that produces a violet-like and woody odor (Müller, 1992). Given that iris/orris appears close to floral, it seems that this descriptor referred to a violet-like odor character. By contrast, orris is close to 
Table 8

\begin{tabular}{|c|c|c|c|c|c|c|}
\hline \multirow[b]{2}{*}{ Category } & \multicolumn{3}{|c|}{ Number of Fragrances } & \multicolumn{3}{|c|}{ Column Percentages } \\
\hline & Women & Men & Unisex & Women & Men & Unisex \\
\hline Fruity & 21 & 0 & 3 & 0.6 & 0.0 & 0.5 \\
\hline Green & 33 & 15 & 29 & 1.0 & 0.9 & 5.3 \\
\hline Water (marine) & 35 & 81 & 21 & 1.0 & 4.7 & 3.8 \\
\hline Floral & 1,446 & 17 & 44 & 41.8 & 1.0 & 8.0 \\
\hline Soft floral & 354 & 10 & 23 & 10.2 & 0.6 & 4.2 \\
\hline Floral oriental & 533 & 1 & 6 & 15.4 & 0.1 & 1.1 \\
\hline Soft oriental & 97 & 18 & 19 & 2.8 & 1.0 & 3.5 \\
\hline Oriental & 145 & 15 & 31 & 4.2 & 0.9 & 5.6 \\
\hline Woody oriental & 352 & 361 & 65 & 10.2 & 21.0 & 11.8 \\
\hline Woods & 71 & 263 & 63 & 2.1 & 15.3 & 11.5 \\
\hline Mossy woods & 175 & 70 & 15 & 5.1 & 4.1 & 2.7 \\
\hline Dry woods & 47 & 156 & 43 & 1.4 & 9.1 & 7.8 \\
\hline Citrus & 146 & 138 & 167 & 4.2 & 8.0 & 30.4 \\
\hline Aromatic (fougère) & 8 & 572 & 21 & 0.2 & 33.3 & 3.8 \\
\hline Total & 3,463 & 1,717 & 550 & 100.0 & 100.0 & 100.0 \\
\hline
\end{tabular}

woody, which might indicate that this attribute referred to a woody-like rather than to a floral odor.

The similarity of leafy, green, and herbaceous (Thiboud, 1991) is reflected in Figure 6 as well as in the loading plot for PC1/PC3 (not shown). The position of green is coincident with Jellinek's odor map (Figure 5), but that is not the case with herbaceous. This term is applied to describe a grassy-green, spicy, and somewhat medicinal odor (Thiboud, 1991), and hence the position of herbaceous in Figure 5 seems more appropriate. This descriptor was applied to 24 materials in Thiboud's database, which is a relatively high number and suggests that herbaceous was used with a different meaning.

Agrestic and rustic are semantically related concepts. Billot (1948) proposed a rustic category of smells that comprised minty, camphoraceous, herbaceous, lavender, green, earthy, and vegetable odors. Hence, rustic could be mapped in the PC1/PC2 plot (Figure 5) by averaging the coordinates of minty, vegetable, lavender, green, and earthy. Interestingly, the resulting position matches nearly exactly with agrestic in Figure 6.

Thiboud's (1991) database contains seven odorants described as minty, four as lavender, and five as camphoraceous. These descriptors share a camphor-like note, but this similarity is not reflected in Figure 6. Their position is not consistent with Figure 5, probably because of the low number of occurrences in the database. Nonetheless, most of the eight descriptors with just four occurrences (cinnamic, rounded, ethereal, musky, leafy, lavender, and iris/ orris) seem to be mapped properly in Figure 6, as already discussed, suggesting that they provide relevant information. Thus, the criterion to discard descriptors with fewer than four occurrences seems appropriate.

\section{Comparison of Results With Edward's Fragrance Wheel}

One perfume category of the Fragrance Wheel is aromatic/fougère. However, given the subjectivity of aro- matic, discussed above, it seems more appropriate to refer to this category as fougère. The Fragrance Wheel was properly rotated so that the floral and woods categories matched approximately the positions of floral and woody, respectively, in Figures 5 and 6. Taking into account that $d r y$ and sweet are opposite concepts in perfumery, $d r y$ woods should appear close to the stimulating corner of the odor effects diagram. This category is next to citrus in the Fragrance Wheel, which corresponds to another corner of the diagram. This observation suggests certain discontinuity between both categories, and we thought that fougère may fit in between. This criterion is discussed below.

We counted the number of men's, women's, and unisex fragrances listed under each category of Edwards's (2008) fragrance guide (Table 8). For a given category, if the percentage of men's perfumes was higher than the percentage of women's, the category was regarded as androgenic (i.e., perceived with masculine characteristics). If the opposite applied (i.e., percent women $>$ percent men, according to Table 8), the category was regarded as gynogenic.

Oriental is a gynogenic category (Table 8), and, moreover, this descriptor is applied more frequently to feminine perfumes (Table 7). Woody oriental is the next category in the wheel, but it is androgenic. Owing to this result, the odor wheel was rotated, so that the boundary between both fragrance categories corresponds to the dashed line in Figures 5 and 7, which can be interpreted as a discriminating line between androgenic versus gynogenic fragrances. Consistent with this interpretation, the three floral categories are the ones more distant from the dashed line and they basically comprise feminine fragrances (Table 8 ). The fact that soft floral is the category most distant from this line is appealing, because soft appears at the upper corner of the odor effects diagram (Figure 7), and, moreover, softening and feminine are related descriptors (Figure 6).

Unisex fragrances are not perceived as clearly masculine or feminine, and hence they may be expected to be 


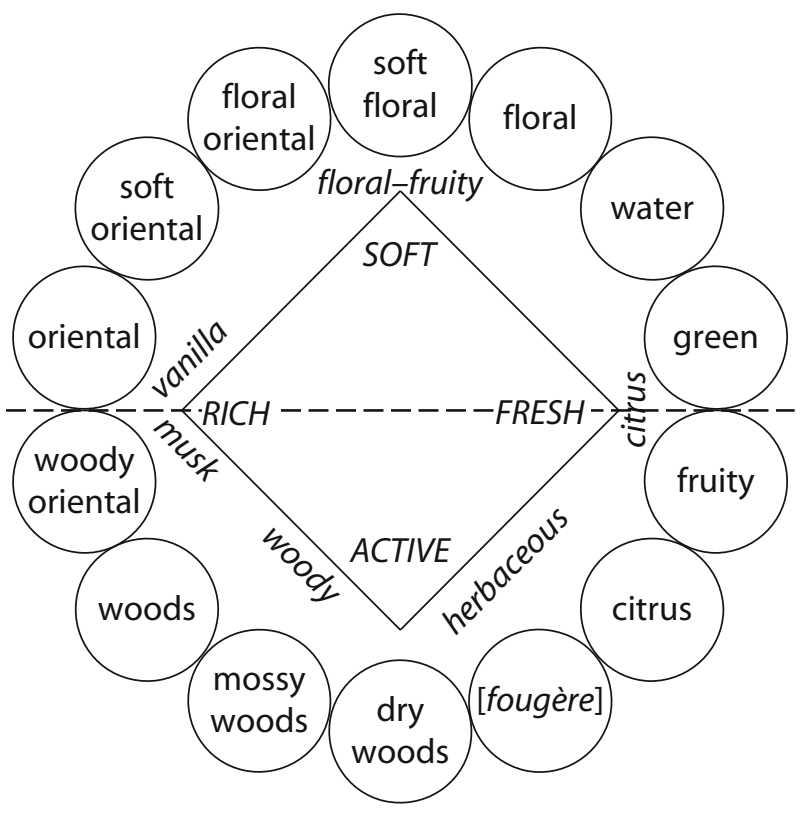

Figure 7. Fragrance Wheel (perfume categories displayed within circles). The sequence of the different categories around the central point is the same as originally was proposed by Edwards (2008), although they were conveniently rotated to achieve the best possible consistency with the odor effects diagram (Calkin \& J. S. Jellinek, 1994), which is also illustrated for comparison purposes (inner square, letters in italics). The only exception is [fougère], which is located at the hub of the odor wheel in the original representation.

found close to the dashed line in Figure 7. Interestingly, woody oriental is the category with the second highest percentage of unisex fragrances (Table 8), and it appears at the boundary of the dashed line. Citrus is a masculine descriptor (Table 6) and an androgenic category (Table 8). Its position in Figure 7 is consistent with Figure 6. Nearly one third of unisex fragrances appear under the citrus category, suggesting that it is also perceived to be close to the boundary between femininity and masculinity.

Fruity is clearly a feminine descriptor (Table 6, Figure 5), and this category contains only feminine or unisex fragrances. Thus, we expected to find it above the dashed line in Figure 7. The fruity category was not included in the 2006 version of the Fragrance Wheel. Edwards (2008) located it between green and citrus, but the proximity of fruity-floral in Figure 5 suggests that the fruity and water categories of fragrances might be swapped in future versions of the wheel. Interestingly, swapping both categories leads to an interesting property of the odor wheel: All categories above the dashed line contain a higher percentage of feminine than of masculine perfumes. The opposite basically applies for categories below the line, with the exception of mossy woods.

According to Edwards (2008), the mossy woods category corresponds to chypre fragrances. We checked that $23.8 \%$ of feminine fragrances in the H\&R guide (Glöss, 1991 ) as well as $35.7 \%$ of masculine fragrances are classified as chypre. This result suggests that mossy woods is an androgenic category, consistent with the position below the dashed line in Figure 7. The fact that mossy and woody are masculine descriptors (Tables 6 and 7; Figure 5) supports this interpretation. Chypre fragrances are characterized by the contrast between a fresh citrus accord and oakmoss (Glöss, 1991). These materials are perceived as basically masculine (Figure 5). Thus, it was unexpected to find that the percentage of women's fragrances in the mossy woods category is slightly higher than that of men's in Edwards's (2008) fragrance guide.

The dry woods family is often called leather, after the dry, smoky scent of Russian leather. It is characterized by dry notes of tobacco and burnt wood (Edwards, 2008). This category is androgenic (Table 8 ) probably because $d r y$, woody, leathery, and tobacco are masculine descriptors (Table 6).

According to Moran (2000), the green category of perfumes is characterized by dominant green notes: vigorous pine, dry herbs (e.g., sage and rosemary), as well as fresh notes from grasses, leaves, lavender, basil, chamomile, and galbanum. The green family of the Fragrance Wheel is characterized by the impact of the classic resinous galbanum accord, which is clearly present in many green fragrances (Edwards, 2008). In the B-H database, galbanum resinoid was the reference for tart (dry). Figure 5 suggests that this descriptor is perceived as masculine, as well as coniferous, vegetable, lavender, and green. These observations suggest that the green category should be regarded as androgenic. But green is used in the H\&R fragrance guide as a feminine descriptor (Table 6). The percentage of men's fragrances under the green category is nearly the same as that of women's (Table 8). Thus, it is somewhat unclear whether green should be categorized as gynogenic or androgenic. Nevertheless, the position of green in Figure 7 is intuitively appealing, because this category appears at the boundary line that discriminates androgenic from gynogenic categories, and because an equivalent position for green in the odor effects diagram was proposed by P. Jellinek (1997).

The fougère category takes its name from the legendary fragrance Fougère Royale, which was created in 1882. In perfumery, fougère is the name of a combination of fresh herbaceous lavender notes on a mossy foundation (Müller, 1992). These materials are basically perceived as masculine (Figure 5), and consequently fougère is an androgenic category (Table 8). Its position could be obtained approximately by averaging the coordinates of fresh, herbaceous, lavender, and mossy in Figure 5. The resulting position is highly coincident with the one that we propose for the fougere category in Figure 7. The twodimensional map of fragrances proposed by J. S. Jellinek (1992) is based on the dimensions warm/cool and florall nonfloral. Taking into account these dimensions, fougère fragrances were mapped in the direction intermediate between cool and nonfloral (J. S. Jellinek, 1992). The same position for the fougère category was proposed in J. S. Jellinek (1990), which is consistent with Figure 7. Floral and fougère are the main categories of feminine and masculine fragrances, respectively. It is appealing that both categories are located at symmetrical positions with respect to the dashed line. 


\section{GENERAL DISCUSSION}

Olfactory perception is based on the activation by odorant molecules of olfactory receptors (ORs) located at the cilia of olfactory neuronal endings (see Zarzo, 2007). Humans exhibit high variability of both general olfactory thresholds and sensitivities toward specific odorantsthat is, partial anosmia (see Lawless, 1997). These phenotypes can be attributed partly to genetic variation in the OR genome. Some OR genes show both functional and inactive alleles in the human population, which are referred to as segregating pseudogenes (SPG). The variability of OR genes among individuals is one of the most pronounced cases of functional population diversity in the human genome. One study found 38 SPGs among the 384 intact human OR genes likely to encode functional ORs (Menashe, Aloni, \& Lancet, 2006). Individualized SPG combinations generate an olfactory barcode, whereby every human nose is genetically different. Thus, the olfactory qualities of complex mixtures, such as fragrances, are not perceived identically by everybody.

Taking into account this intersubject olfactory variability, and given the high dimensionality of odor perception space, it was rather unexpected to find that the multivariate analysis of two odor profile databases obtained in the context of perfumery led to rather coincident twodimensional representations of the odor descriptor space (Figures 5 and 6). Moreover, these odor maps are highly consistent with other studies (e.g., Thiboud, 1991) as well as with odor representations such as Jellinek's odor map and Edwards's Fragrance Wheel, which are derived basically from the experience of perfumers. These results encourage further attempts to achieve standard odor maps for perfumery. Moreover, the reported evidence supports the hypothesis that, apart from individual exceptions, the effect of a given odorous material is basically the same for all people if the odor is perceived under a similar context and concentration (P. Jellinek, 1997).

Other authors have applied multivariate statistical methods such as PCA, factor analysis, or cluster analysis to the B-H database in order to classify compounds into groups with similar odor aspect patterns (Boelens \& Haring, 1981; Ennis et al., 1982). Other studies have used this database in an effort to relate molecular structure with odor character (Palen, 1983; Seeman, Palen, \& Ennis, 1987). None of these studies has attempted to interpret the correlation structures among odor attributes. Our work is the first study showing that PC1 and PC2 of this database are consistent with the odor effects diagram.

Jellinek's odor map is based on two primary dimensions of odor effects: erogenous versus antierogenous (refreshing) and narcotic versus stimulating. The former dimension corresponds to $\mathrm{PC} 1$ of the $\mathrm{B}-\mathrm{H}$ database, because fresh is the descriptor with highest $p$ [1] loading. P. Jellinek (1997) assumed that erogenous and refreshing were opposite polarities of the same underlying dimension. But erogenous and animal determine an independent dimension (Figure 3) that is orthogonal to PC1. Thus, PC1 seems better interpreted with the polarity refreshing versus warming, or maybe as a psychological dimen- sion that evokes natural environments associated with the presence of water versus dry environments. The narcoticl stimulating dimension of the odor effects diagram corresponds to PC2, which basically accounts for the polarity feminine/masculine as suggested by P. Jellinek (1997). The results of the $\mathrm{B}-\mathrm{H}$ database are intuitively appealing, because most of the 30 references are raw materials commonly used to formulate perfumes. Moreover, this database was obtained from a panel of perfumers, and their expertise is probably heavily imprinted in the resulting perceptual space. The fact that a similar odor map was obtained from the analysis of Thiboud's database supports the hypothesis that the two dimensions of the odor effects diagram are the most salient in the analysis of cosmetic scents. The consistency among odor maps provides clues to explain why some descriptors were mapped in different relative positions.

Although the PC1/PC2 plot from the $\mathrm{B}-\mathrm{H}$ database is rather similar to the one from Thiboud's (1991) database, the former seems more reliable. Actually, we were able to interpret all of the discrepancies found between this plot and Jellinek's odor map, but this was not the case with Thiboud's database. Moreover, our results suggest that the $\mathrm{B}-\mathrm{H}$ database contains more information to identify further relevant dimensions. The main disadvantage of numeric methods is that they are time consuming, and hence they are usually restricted to a limited number of descriptors. By contrast, semantic methods allow for a rapid generation of data and permit a larger number of attributes, which is a great advantage with respect to interpreting the psychological aspects involved in olfaction. The approach followed in the present work, combining the information from numeric and semantic profiles in order to characterize odor descriptor space, appears to be a good alternative. The database obtained by Dravnieks (1985) is relevant to olfactory research, because it contains numeric odor profiles based on 146 descriptors. It would be of interest to study whether the basic dimensions of odor effects would also be salient in a multivariate analysis of this database.

All attempts to categorize odorants or odor descriptors on the basis of the analysis of odor profile databases are obviously limited by the finite set of chemical stimuli that participants evaluate. Taking into account that the perfumer's palette now consists of approximately 4,000 raw materials, it is necessary to determine the most appropriate sample size to assess. Obviously, using a large number of materials is more likely to better represent the whole population, but this would be restricted to semantic methods. The sensory study reported by Higuchi et al. (2004) using 11 odorant materials found three relevant factors, which the authors interperted as clarity (i.e., freshness), softness (i.e., femininity), and intensity. Their results suggest that the basic dimensions of the odor-effects diagram are salient even with a reduced number of materials. Similar results were reported by others using only 10 fragrances (J. S. Jellinek, 1990, 1992; J. S. Jellinek et al., 1992), but this number is obviously too low to achieve a detailed map of odor descriptors. The fact that consistent results were obtained from Thiboud's (1991) database, with a sample size of 119 materials, suggests that it is unnecessary to as- 
sess many hundreds of compounds. Conversely, it seems more convenient to focus on the psychophysical method of odor description. Nonetheless, some descriptors such as minty and lavender were discordantly mapped, probably because the database lacked enough representative odorants with a camphoraceous note.

Our analysis of the B-H database also revealed that the resulting odor representation was affected strongly by the materials chosen as a reference for each descriptor. Thus, in order to guarantee consensus in the development of sensory maps for cosmetic odor descriptors, it is necessary to first achieve certain agreement among perfumers about the reference materials to be used for each attribute. The use of references is strongly recommended, because each individual's preconceptions about a given odor descriptor are not necessarily the same as another's. Perfumers think about odors in terms of the odors' use in a fragrance. Selecting the right reference material at the right concentration for each odor descriptor is important for reproducibility purposes, given that the similarities between attributes are partly determined by the odor similarities of the reference materials. Harper (1975) provides a list of reference materials chosen by a group of experts for 44 descriptors. The International Organization for Standardization (ISO) proposes 24 reference substances for training in the recognition of odors (ISO, 2006). Further efforts will be necessary in order to reach a consensus about which standards should be used for the basic attributes in the description of perfumery odors.

Some olfactory researchers suggest that it is an oversimplification to attempt to reduce the high number of odor qualities to a few dimensions (Lawless, 1997). However, this view is not shared by perfumers, who consider odor maps to be a valuable tool (J. S. Jellinek, 1992). One of the world's most comprehensive guides to commercial fragrances is the one developed by Edwards (2008). It classifies more than 5,700 mass-market and direct-sale fragrances available worldwide, cross-referenced by gender (i.e., women, men, or unisex) and by fragrance families that are displayed on the Fragrance Wheel. This sensory wheel was created in order to simplify fragrance classification as well as to show the relationships among each of the individual families. We compared the Fragrance Wheel with Jellinek's odor map as well as with the resulting odor maps from the two databases analyzed (Figures 5 and 6), and we observed a remarkable coincidence (shown in Figure 7). One of the practical outcomes of the present work is that we propose some modifications of the Fragrance Wheel so that it can be more consistent with other studies. Work in progress suggests that the Discodor (Harder, 1979) is rather consistent with the odor effects diagram. By contrast, the Natural Perfume Wheel (Aftelier, 2006) should be modified significantly in order to reproduce properly the underlying dimensions of odor perception space.

\section{AUTHOR NOTE}

M.Z. is grateful for a postdoctoral grant sponsored jointly by the Fulbright Program and the Spanish Ministry of Education and Science,
State Secretariat of Universities and Research. Sponsorship from Procter $\&$ Gamble Co. (Corporate Modeling and Simulations) is also acknowledged. We thank B. Murch for valuable discussion and comments. Address correspondence to M. Zarzo, Department of Applied Statistics, Universidad Politécnica de Valencia, Camino de Vera s/n, 46022 Valencia, Spain (e-mail: mazarcas@eio.upv.es).

Note-Accepted by the previous editorial team, when Thomas H. Carr was Editor.

\section{REFERENCES}

Abe, H., Kanaya, S., Komukai, T., Takahashi, Y., \& SASAKI, S. (1990). Systematization of semantic descriptions of odors. Analytica Chimica Acta, 239, 73-85. doi:10.1016/S0003-2670(00)83837-4

Aftel, M. (2001). Essence and alchemy: A book of perfume. New York: North Point Press.

Aftelier (2006). Natural perfume wheel. Berkeley, CA: Aftelier Perfumes Inc.

Arctander, S. (1969). Perfume and flavor chemicals (aroma chemicals). Montclair, NJ: Author.

BACIS (2001). Database of perfumery materials and performance (PMP-2001). Huizen, The Netherlands: BACIS.

BAIN, A. (1855). The senses and the intellect. London: Parker.

Billot, M. (1948). Classification des odeurs. Industries de la Parfumerie, 3, 87-92.

Blumenthal, P. (1979). Geruchsbezeichnungen in der französischen werbesprache. Vox Romanica, 38, 155-173.

Boelens, H., \& Haring, H. G. (1981). Molecular structure and olfactive quality [Internal report]. Bussum, The Netherlands: Naarden International.

Brechbill, G. O. (2007). Classifying aroma chemicals. Creative Endeavor Books. Available at www.perfumerbook.com.

BRUD, W. S. (1986). Words versus odors: How perfumers communicate. Perfumer \& Flavorist, 11, 27-44.

BuRDOCK, G. A. (ED.) (2004). Fenaroli's handbook of flavor ingredients (5th ed.). Boca Raton, FL: CRC Press.

Calkin, R. R., \& Jellinek, J. S. (1994). Perfumery: Practice and principles (1st ed.). New York: Wiley.

Callegari, P., Rouault, J., \& Laffort, P. (1997). Olfactory quality: From descriptor profiles to similarities. Chemical Senses, 22, 1-8. doi:10.1093/chemse/22.1.1

Chastrette, M. (1998). Data management in olfaction studies. SAR \& QSAR in Environmental Research, 8, 157-181. doi:10.1080/10629369808039139

Chastrette, M., de Saint Laumer, J.-Y., \& Sauvegrain, P. (1991). Analysis of a system of description of odors by means of four different multivariate statistical methods. Chemical Senses, 16, 81-93. doi:10.1093/chemse/16.1.81

Chastrette, M., Elmouaffek, A., \& Sauvegrain, P. (1988). A multidimensional statistical study of similarities between 74 notes used in perfumery. Chemical Senses, 13, 295-305. doi:10.1093/ chemse/13.2.295

Coxon, J. M., Gregson, R. A. M., \& Paddick, R. G. (1978). Multidimensional scaling of perceived odour of bicyclo[2.2.1] heptane, 1,7,7-trimethylbicyclo[2.2.1] heptane and cyclohexane derivatives. Chemical Senses \& Flavor, 3, 431-441. doi:10.1093/chemse/3.4.431

DAVIS, R. G. (1979). Olfactory perceptual space models compared by quantitative methods. Chemical Senses, 4, 21-33. doi:10.1093/ chemse/4.1.21

DRAVNIEKS, A. (1985). Atlas of odor character profiles, data series DS 61. Philadelphia: American Society for Testing and Materials.

Edwards, M. (2006). Fragrances of the world: Parfums du monde 2006 (22nd ed.). La Quinta, CA: Crescent House.

EDWARDS, M. (2008). Fragrances of the world: Parfums du monde 2008 (24th ed.). Sydney: Fragrances of the World.

Ennis, D. M., Boelens, H., Haring, H., \& Bowman, P. (1982). Multivariate analysis in sensory evaluation. Food Technology, 36, 83-90.

GLöss, W. (ED.) (1991). H\&R fragrance guide: Feminine notes, masculine notes (2nd ed.). Hamburg: Glöss Verlag.

Green, M. (1999). Natural perfumes: Simple aromatherapy recipes. Loveland, CO: Interweave Press. 
HARDER, U. (1979). Der H\&R duftkreis. Haarman \& Reimer Contact, 23, 18-27.

HARPER, R. (1975). Some chemicals representing particular odour qualities. Chemical Senses \& Flavor, 1, 353-357. doi:10.1093/ chemse/1.3.353

Higuchi, T., ShoJI, K., \& Hatayama, T. (2004). Multidimensional scaling of fragrances: A comparison between the verbal and non-verbal methods of classifying fragrances. Japanese Psychological Research, 46, 10-19. doi:10.1111/j.1468-5884.2004.00232.x

Hummel, T., \& Livermore, A. (2002). Intranasal chemosensory function of the trigeminal nerve and aspects of its relation to olfaction. International Archives of Occupational \& Environmental Health, 75, 305-313. doi:10.1007/s00420-002-0315-7

InTERNATIONAL ORganization For STANDARDiZation (2006). Sensory analysis-Methodology —Initiation and training of assessors in the detection and recognition of odors (ISO 5496). Geneva: International Organization for Standardization.

Jaubert, J. N., TAPiero, C., \& Doré, J. C. (1995). The field of odors: Toward a universal language for odor relationships. Perfumer \& Flavorist, 20, 1-16.

JELLINEK, J. S. (1980). Fragrance character-The consumer's viewpoint. Dragoco Report, 27, 19-24.

JELLINEK, J. S. (1990). A consumer oriented classification of perfumes. Dragoco Report, 37, 16-29.

JellineK, J. S. (1992). Perfume classification: A new approach. In S. Van Toller \& G. H. Dodd (Eds.), Fragrance: The psychology and biology of perfume (pp. 229-242). London: Chapman \& Hall.

JellineK, J. S. (1994). Aroma-chology: A status review. Perfumer \& Flavorist, $19,25-49$.

JELLINEK, J. S. (1997). The psychological basis of perfumery: A reevaluation. In J. S. Jellinek (Ed.), The psychological basis of perfumery (4th ed., pp. 240-256). London: Chapman \& Hall.

Jellinek, J. S., Du Bosque, B. O., Gschwind, J., Schubert, B., \& SCHARF, A. (1992). The scent and the marketing mix. Dragoco Report, 39, 103-121.

JellineK, P. (1951). Die psychologischen Grundlagen der Parfümerie. Heidelberg: Alfred Hüthig Verlag.

JELLINEK, P. (1997). The psychological basis of perfumery. In J. S. Jellinek (Ed.), The psychological basis of perfumery (4th ed., pp. 1-162). London: Chapman \& Hall.

Jeltema, M. A., \& Southwick, E. W. (1986). Evaluation and applications of odor profiling. Journal of Sensory Studies, 1, 123-136. doi:10.1111/j.1745-459X.1986.tb00165.x

Jennings-White, C. (1984). Human primary odors. Perfumer \& Flavorist, 9 , 46-58.

Kirk-Smith, M. D., \& Booth, D. A. (1987). Chemoreception in human behaviour: Experimental analysis of the social effects of fragrances. Chemical Senses, 12, 159-166. doi:10.1093/chemse/12.1.159

KLeIN, S. (1947). Primary odor element classification. American Perfumer \& Essential Oil Review, 50, 453-454.

LAWLESS, H. T. (1997). Olfactory psychophysics. In G. K. Beauchamp \& L. Bartoshuk (Eds.), Tasting and smelling (pp. 125-174). San Diego: Academic Press.

Madany-Mamlouk, A., Chee-Ruiter, C., Hofmann, U. G., \& Bower, J. M. (2003). Quantifying olfactory perception: Mapping olfactory perception space by using multidimensional scaling and self-organizing maps. Neurocomputing, 52-54, 591-597. doi:10.1016/ S0925-2312(02)00805-6

Menashe, I., Aloni, R., \& Lancet, D. (2006). A probabilistic classifier for olfactory receptor pseudogenes. BMC Bioinformatics, 7, 393. doi:10.1186/1471-2105-7-393

Moran, J. (2000). Fabulous fragrances II: A guide to prestige perfumes for women and men. La Quinta, CA: Crescent House Publishing.
MÜLLER, J. (1992). The H\&R book of perfume: Understanding fragrance; origins, history, development; guide to fragrance ingredients. Hamburg: Glöss Verlag.

Nute, G. R., Macfie, H. J. H., \& Greenhoff, K. (1988). Practical application of preference mapping. In D. M. H. Thomson (Ed.), Food acceptability (pp. 377-386). London: Elsevier.

Palen, J. (1983). The Boelens odor profiling study: Analyses and evaluations of odor/flavor profiling. Experiments and suggestions for future studies [Internal report]. Philip Morris USA. Retrieved from www .pmdocs.com.

Pintore, M., Wechman, C., Sicard, G., Chastrette, M., Amaury, N., \& Chretien, J. R. (2006). Comparing the information content of two large olfactory databases. Journal of Chemical Information \& Modeling, 46, 32-38. doi:10.1021/ci0502505

Poucher, W. A. (1974). Perfumes, cosmetics and soaps: Vol. 1. The raw materials of perfumery. London: Chapman \& Hall.

Richardison, J. T., \& Zucco, G. M. (1989). Cognition and olfaction: A review. Psychological Bulletin, 105, 352-360. doi:10.1037/0033 $-2909.105 .3 .352$

Rimmel, E. (1895). The base of perfumes. London.

SChiffMAn, S. S. (1974). Physicochemical correlates of olfactory quality. Science, 185, 112-117. doi:10.1126/science.185.4146.112

ScHUTZ, H. G. (1964). A matching-standards method for characterizing odor qualities. Annals of the New York Academy of Sciences, 116, 517526. doi:10.1111/j.1749-6632.1964.tb45081.x

Seeman, J. I., Palen, J., \& EnNis, D. M. (1987). Prediction of the odor quality of organic compounds: Odor profiling and chemical structure correlations [Internal report]. Philip Morris USA. Retrieved from www.pmdocs.com.

Sigma-Aldrich Fine Chemicals Company (2003). Flavors and fragrances 2003-2004 catalog. Milwaukee, WI: Author.

Stevenson, R. J., Prescott, J., \& Boakes, R. A. (1995). The acquisition of taste properties by odors. Learning \& Motivation, 26, 1-23. doi:10.1016/S0023-9690(05)80006-2

ThiBoud, M. (1991). Empirical classification of odours. In P. M. Müller \& D. Lamparsky (Eds.), Perfumes: Art, science, and technology (pp. 253-286). New York: Elsevier.

Tisserand, R. (1988). Essential oils as psychotherapeutic agents. In S. Van Toller \& G. H. Dodd (Eds.), Perfumery: The psychology and biology of fragrance (pp. 167-181). London: Chapman \& Hall.

Wise, P. M., Olsson, M. J., \& CAIn, W. S. (2000). Quantification of odor quality. Chemical Senses, 25, 429-443. doi:10.1093/ chemse/25.4.429

Yoshida, M. (1975). Psychometric classification of odors. Chemical Senses \& Flavor, 1, 443-464. doi:10.1093/chemse/1.4.443

Zarzo, M. (2007). The sense of smell: Molecular basis of odorant recognition. Biological Reviews, 82, 455-479. doi:10.1111/j.1469 $-185 X .2007 .00019 . \mathrm{X}$

Zarzo, M. (2008a). Psychologic dimensions in the perception of everyday odors: Pleasantness and edibility. Journal of Sensory Studies, 23, 354-376. doi:10.1111/j.1745-459X.2008.00160.x

Zarzo, M. (2008b). Relevant psychological dimensions in the perceptual space of perfumery odors. Food Quality \& Preference, 19, 315322. doi:10.1016/j.foodqual.2007.10.007

Zarzo, M., \& Stanton, D. T. (2006). Identification of latent variables in a semantic odor profile database using principal component analysis. Chemical Senses, 31, 713-724. doi:10.1093/chemse/bj1013

ZWAardemaker, H. (1925). Classification des odeurs. In L'odorat (pp. 178-224). Paris: Doin.

(Manuscript received December 28, 2007; revision accepted for publication September 9, 2008.) 